QA
501
W24

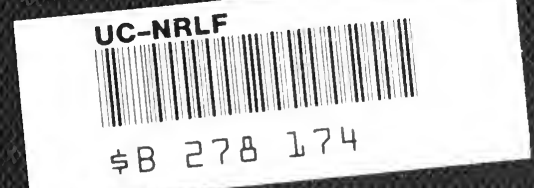

\title{
Manual of
}

Descriptive Creometry

m

$\underline{-}$

$r$

$\infty$ 


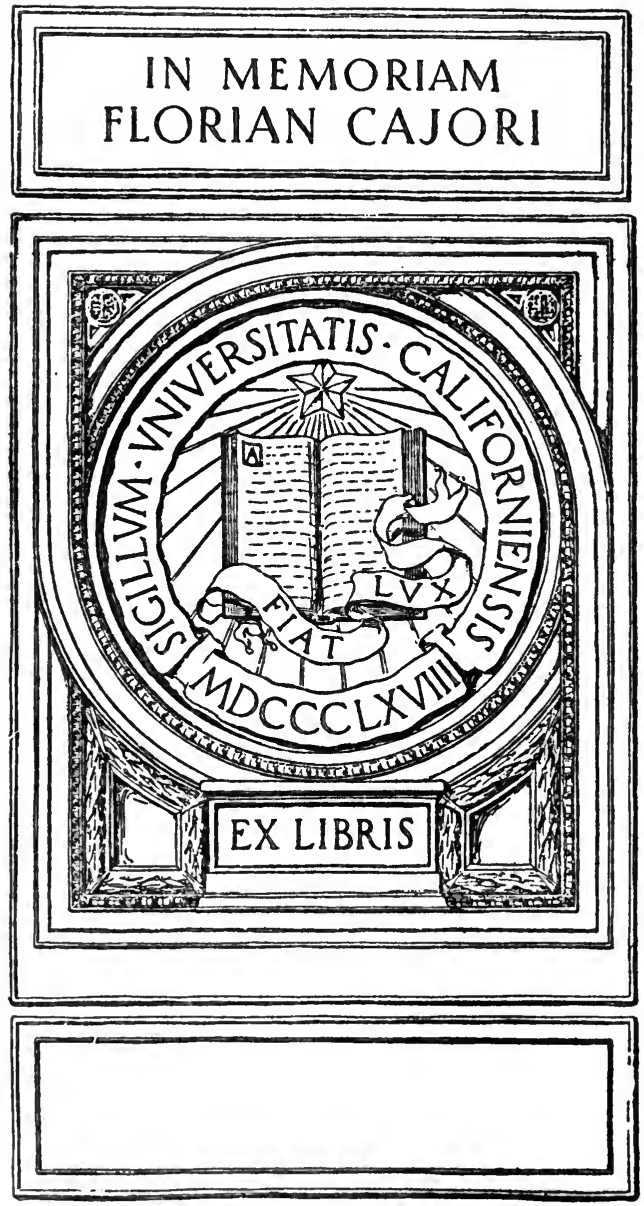


Flovian Cajori. 
Digitized by the Internet Archive in 2007 with funding from Microsoft Corporation 


\section{A MANUAL}

\section{or \\ DESCRIPTIVE GEOMETRY,}

WITH

\section{NUMEROUS PROBLEMS.}

$\alpha^{3}$

BY

CLARENCE A. WALDO, A.M.,

Profissor of Mathematics, Purdue University, LAFAYETTE, IND.

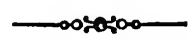

\section{BOSTON:}

PUBLISHED BY D. C. HEATH \& CO.

1895. 
Copyright, 1887, By ClARENCE A. WALDO.

J. S. Cushing \& Co., Printers, Boston. 


\section{PREFACE.}



$\mathrm{D}$ ESCRIPTIVE GEOMETRY gives power to express conceptions and to solve problems in the constructive arts; it also effectively disciplines the geometrical imagination.

To accomplish these ends, nothing is better than problems of progressive difficulty, which, taken in their logical order, the student can master alone, or with the aid of a small amount of judicious suggestion, and this principle has controlled the plan of this book. Part I., therefore, consists exclusively of problems systematically arranged. The Introduction should be read before the student undertakes to solve these, as it is not intended for recitation, but for a preparatory lecture and for reference. It is not expected that any student will solve all the problems, nor wonld it be a wise expenditure of time. A course has been laid down, - by no means a minimum one, and in Part II. of the book, suggestions, analyses, and occasional demonstrations for the solution of the problems of this course have been given, with the intention, however, of always leaving some real work for the student. A large number of additional problems have been stated, which can be substituted at will for the others or can be used independently. Such combinations of the problems can be readily formed that the instructor may have from year to year the substantial advantages of a change of text-book. 
Part III. is a condensed statement designed for occasional reference in the earlier part of the work, but especially as a review before leaving the subject or in preparing for examination.

In using the material provided in this book, the author has found a method somewhat as follows productive of the best results: Out of every three exercises, one, an hour in length, is spent with the class in explaining difficulties, in opening new phases of the subject, and in pointing out short and elegant methods of solution, based as far as possible upon the discoveries of the class. As often as seems necessary, analyses and geometrical reasons are called for. The other two exercises, each two hours in length, are spent by the students in work under the eye of the instructor, in solving and reporting problems, and receiving such assistance as seems necessary or judicious.

When the constructions have been approved, the student copies and arranges them, and prepares a suitable index and title-page. The set of solutions thus formed is then permanently bound, and in the end becomes the property of the student who makes it.

The following are the special features of the book:

First. The method of unfolding the subject by problems systematically arranged, and supplemented by suggestions when needed.

Second. The large number of problems given.

Third. The method of stating the problems, which in connection with the notation adopted makes every lettered drawing entirely self-explanatory.

Fourth. The introduction of several subjects of considerable descriptive value, such as the axis of affinity, axonometry, Pascal's and Brianchon's hexagrams. 
Fifth. The early discussion of the cone and cylinder of revolution, and the sphere, in order that from the beginning these surfaces may be used as auxiliary.

Sixth. The omission of all plates except a few of a generic character.

It has been the intention of the author to prepare a book that will stimulate the student and can safely be left in his hands at all times, in the same way that a book of directions may be left in the hands of a student in a zoölogical laboratory. It is hoped that any one of three classes of teachers of the subject will find it serviceable:

First. Those who believe it necessary to continue the methods of demonstration peculiar to Ancient Geometry through the course in Descriptive Geometry, but wish to supplement this work with practical exercises.

Second. Those who prefer the lecture system rather than the use of text-books, but desire a book of exercises for the systematic grounding of their students in the elements.

Third. Those who try to find in these pages all they need for a short, thorough course in the fundamental principles of Descriptive Geometry.

The book is intended for the class-room, but it is believed that the industrious student will be able to master it by himself.

Several books in German have been freely drawn upon for problems, though many of them were collected while the author was attending a course of lectures upon the subject, by Professor Marx, of the Royal Polytechnic School at Munich, and some are entirely original. All, however, have been rearranged and recast to suit the requirements of the present work. Pohlke has been freely consulted in the preparation of 
Part III., though the works of De la Gournerie, Mannheim, Delabar, Gugler, Fiedler, Steiner and others have been at hand for reference.

I wish to express my thanks to Pres. T. C. Mendenhall, of the Rose Polytechnic Institute, and to my associate in the Faculty, Prof. W. L. Ames, of the department of Mechanical Drawing, both of whom read my manuscript and made valuable and helpful suggestions; also to Mr. E. G. Waters, a student of Rose Polytechnic, who has aided me in the preparation of the plates for this work.

C. A. WALDO.

Terre HaUte, IND.,

June 17, 1887. 


\section{TABLE OF CONTENTS.}

\section{INTRODUCTION.}

The purpose and place of Descriptive Geometry $\quad$. $\quad . \quad$. $\quad \begin{array}{r}\text { PAGE } \\ 1\end{array}$

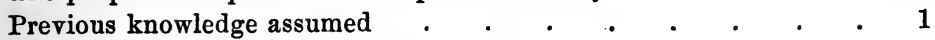

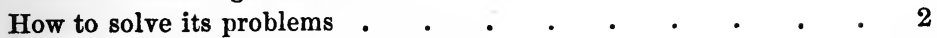

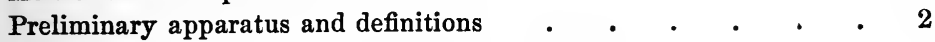
The projection of a point, line, surface, or solid $\quad$. $\quad . \quad$. $\quad 3$ Transformation of fundamental planes . . . . . . . 4 Discussion of a preliminary exercise • . . . . . . 4 Recapitulation • . . . • . • • • • • 6

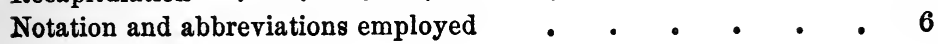

\section{PART I.}

\section{EXERCISES AND PROBLEMS.}

\section{Section I.}

Point, Line and Plane.

PROBLEMS.

1-2. Representation of a point . . . . . . . 11

3-10. Construction of right lines $\quad$. $\quad$. $\quad$. $\quad$. $\quad$. $\quad$. 11

11-12. Description of cone and cylinder of revolution and sphere 12

13-20. Right line. • . . . . . . • . 12

21. Point and right line . . . . . . . . . 13

24-30. Plane. . . . . . . . . . . . 13

23, 31-36. Plane and point therein . . . . . . . . 13

22, 37. Plane and line therein . . . . . . . . $\quad . \quad 13$

38,39 . Two intersecting lines in a plane . . . . . . 13

40. Two parallel lines in a plane . . . . . . . $\quad . \quad$. 14

41-45. Point and right line in the plane . . . . . . 14 
PROBLEMS.

46, 47. Plane and point without it

48. Plane and right line without it . . . . . 14

49-53. Plane and point and line without it . . . . . 14

54, 55. Two intersecting planes . . . . . . . . . 15

56. Two parallel planes . . . . . . . . . . . $\quad . \quad 15$

57-60. Plane and non-intersecting lines in space . . $\quad 15$

61-66. Two planes, point and line . . . . . . $\quad$. 15

67-74. Trihedral angle . . . . . . . . . 16

75, 77. Bounded plane figure . . . . . . . . 16

76. Axis of affinity . . . . . . . . . . 16

78-85. Pyramid . . . . . . . . . . . 16

86,87. Regular polyhedrons . . . . . . . . $\quad . \quad 17$

88-91. Plane section of polyhedrons . $. \quad . \quad . \quad . \quad . \quad 18$

92, 93. Intersection of right line and polyhedrons . . . 18

94-96. Intersections of polyhedrons . . . . . . 18

97-104. Axonometry . . . . . . . . . 18

\section{Section II.}

Additional exercises on the Point, Line and Plane

\section{Section III.}

Lines and Surfaces of an Order Higher than the First.

171-178. Lines of the second order . . . . . . $\quad 25$

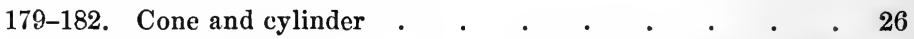

183-186. Development of cone and cylinder, together with plane section . . . . . . . . . . . 26

187-189. Piercing of cone, cylinder and sphere . . . . $\quad .27$

190-201. Tangent plane to cone, cylinder and developable surface 27

202-207. Intersection of solids, at least one in each case being

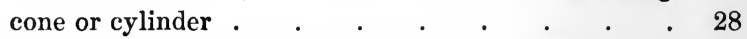

208-214. Tangent plane to surfaces of revolution . . . . 28

215. Tangent cone to ellipsoid . . . . . . . $\quad$. 29

216,217 . Surfaces of revolution intersected by solids . $\quad . \quad$. 29

218-224. Warped surfaces . . . . . . . . . 29

\section{Section IV.}

Additional exercises on Lines and Surfaces of an Order Higher than the First 


\section{PART II.}

SUGGESTIONS, ANALYSES, AND DEMONSTRATIONS.

Section I.

Point, Line and Plane.

Problems 1-104 • . . . . . . . . . . . . 36

Axis of affinity defined and its existence demonstrated . . . $\quad 45$

Axonometry . . . . . . . . . . . 50

Section II.

Lines and Surfaces of an Order Higher than the First.

Problems 171-223

Conic section inscribed in a parallelogram as the locus of a system of points . . . . . . . . . . . . .

Conic section inscribed in a parallelogram as the envelope of a sys-

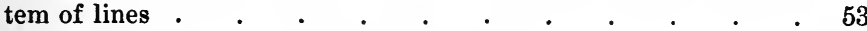

Pascal's hexagram $\quad . \quad$. $\quad . \quad . \quad . \quad . \quad . \quad . \quad . \quad 55$

Brianchon's hexagram . . . . . . . . . . $\quad$. 57

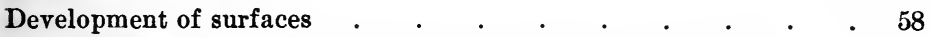

Demonstration for the hyperboloid of revolution of one sheet . $\quad 61$

\section{PART III.}

SUMMARY OF PRINCIPLES AND DEFINITIONS.

\section{Section I.}

Projections.

ARTICLE.

1. Descriptive Geometry . . . . . . . 65

2. Shading and perspective. . . . . . . . . 65

3. Representation of a solid . . . . . . . . . . . 65

4. Central projection . . . . . . . . . . 65

5. Parallel projection . . . . . . . . . . . . . 65

6. Oblique parallel projection . . . . . . . . . 65

7. Orthogonal parallel projection . . . . . . . . 66

8. Restricted meaning of Descriptive Geometry . . . . 66

9. Planes of projection. $. \quad . \quad . \quad . \quad . \quad . \quad . \quad .666$

10. Ground line . . . . . . . . . . . . . 66

11. Revolutions . . . . . . . . . . . . . 66 
Section II.

Point, Line and Plane.

ARTICLE.

12. Projection of a point

PAGE

13. Projection of a right line. $. \quad . \quad . \quad . \quad . \quad . \quad .67$

14, 15. Traces of a line

16. Angle of inclination .

17. Projection of parallel right lines

18. Plane, how represented . . . . . . . . . . . 67

19. Plane, how determined . . . . . . . . . . 67

20. Point in plane . . . . . . . . . . . . . 67

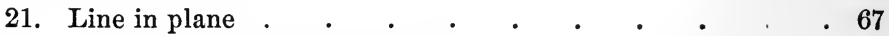

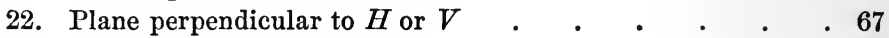

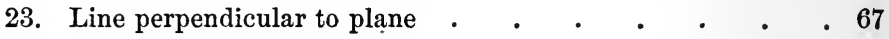

24. Two parallel planes . . . • . • . . . 68

\section{Section III.}

Line in General.



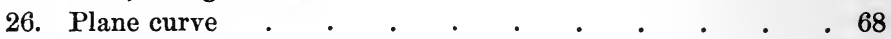

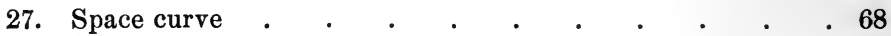

28. Classification of plane curves . $\quad . \quad$. $\quad . \quad$. $\quad .68$

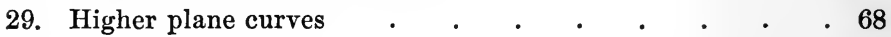

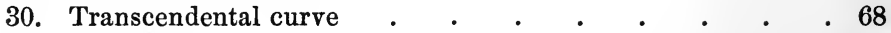

31. Tangent . . . . . . . . . . .

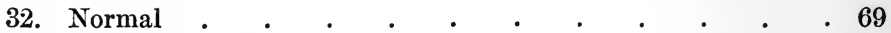

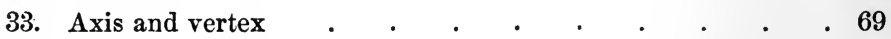

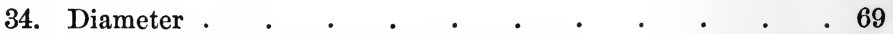

35. Osculating circle and radius of curvature $\quad$. $\quad . \quad 69$

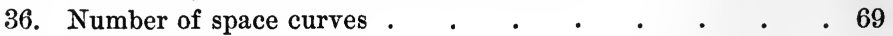

37. Helix . . . . . . . . . . . . .

38. Spherical epicycloid . . . . . . . 69

\section{Section IV.}

Surfaces in General.

39. Surface

40. Directrix, directer

41. Kinds of surfaces 
$\triangle$ BTTCLE.

42. Algebraical surfaces . . . . . . . . . 70

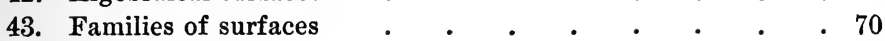

44. Ruled surfaces . • . . . . . . . . 70

45. Double curved surfaces . . . . . . . . . 71

Section V.

Developable Surfaces.

46. Developable surface further defined . . . . . 71

47. Developable surface with helical directrix . . . . 71

48. Special developable surface . . . . . . . . 71

49. Cone. . . . . . . . . . . . . . 72

50. Cylinder . . . . . . . . . . . .

51. Tangent plane . . . . . . . . . . . . 72

52. Shortest path . . . . . . . . . . 72

\section{Section VI.}

Surfaces of Revolution.



54. Axis . . . . . . . . . . . . . . . 72

55. Parallels . . . . . . . . . . . . 73

56. Meridian . . . . . . . . . . . $\quad$. 73

57. Equator . . . . . . . . . . . . 73

58. Representation of surfaces of revolution. . . . . 73

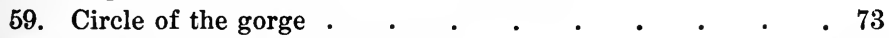

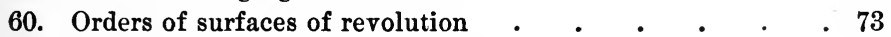

61. Subdivision of surfaces of revolution $\quad$. $\quad . \quad$. $\quad . \quad$. 73

62. Tangent plane . . . . . . . . . . . . $\quad$. 74

\section{Section VII.}

Warped Surfaces.

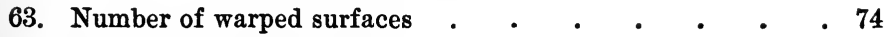

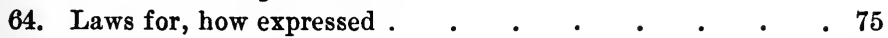

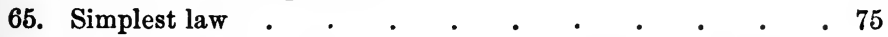

66. Property of a plane containing an element of a warped surface . . . . . . . . . . . 75

67. Mutually tangent warped surfaces . . . . . . $\quad$. 75

68. Divisions of warped surfaces . . . . . . . 76 
xii

TABLE OF CONTENTS.

ARTICLE.

69. Infinite directrices.

70. But one infinite directrix . .

71. Special group of warped surfaces . $. \quad . \quad . \quad . \quad .77$

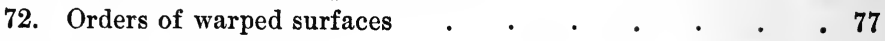

73. Higher orders . . . . . . . . . . . . $\quad$. 77

74. Screw surfaces . $. \quad . \quad . \quad . \quad . \quad . \quad . \quad . \quad$. 77 


\section{INTRODUCTION.}

How can a solid having three dimensions be exactly represented upon a surface having but two dimensions ?

This is the problem which Descriptive Geometry seeks to answer. As the theoretical basis of its answer it develops certain laws of relationship which connect the figure in space with its expression in a plane. These laws belong to Projective Geometry and are rigorously mathematical; when, however, actual representations of real objects are attempted, the results will be approximations of varying degrees of accuracy according to the skill of the artist. Descriptive Geometry is an art when it exercises a student in its methods; a science, when it reveals a strictly mathematical basis for its methods.

To the technologist, as the architect or mechanic, it is not only necessary that the representation should be derived from the original and suggest it in a general way, but it is even more imperative that the original itself, which may have been a material object or only a creation of the imagination, may be reproduced by the skilled workman with the aid of the representation in tangible, material form, in every smallest detail of shape and measurement. Because rectangular or orthographic projection accomplishes this twofold object best, it has generally been allowed to usurp the whole domain of Descriptive Geometry, and it is not the purpose of this little book to depart greatly from the usual though inadequate interpretation of the science. For the sake of special descriptive properties easily understood, the more general science of Projective Geometry is drawn upon for a $\mathrm{few}$ isolated propositions.

A knowledge of plane and solid elementary or ancient Geometry is assumed. Especial attention, however, is directed to 
those propositions which in most American manuals are embraced in the first two books of solid Geometry. The student is also supposed to have a fair knowledge of elementary Algebra and Trigonometry; and in that portion of this work which treats of figures of a higher order than the first, of elementary Analytic Geometry.

When statements without demonstration are made, it is with the expectation that the student will think them through and satisfy himself that they are founded in formal Geometry or in common sense. He should hold in mind the figures presented to him for consideration until in imagination he can see them in their true forms and relations. He will then be able to perform operations upon them as upon material objects presented to the senses. This is the essence of Geometry. No exercise of the mathematical faculties can be more productive of beneficial results, and there is no other that will give a more pleasurable feeling of mastery.

Preparatory to the solution of the problems let us now consider

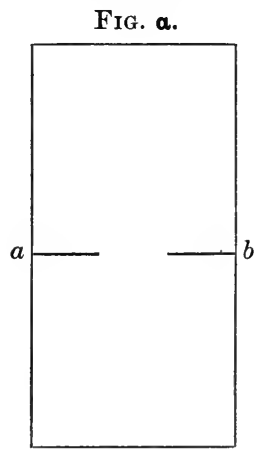
in a practical way the meaning of orthographic projection and discuss briefly the transformations usually made. For this purpose let us take a piece of stiff writingpaper about eight inches long and four wide and cut it as in Fig. $a$, - two slits along the medial line $a b$, about one inch into each side.

Take another piece of paper of the same size cutting it as in Fig. $\beta,-a$ two-inch slit in the line $a b$, leaving an inch on each side of the paper uncut. By folding over the top of a without creasing it we will thrust it through the slit of $\beta$. The two pieces will then hinge along their medial lines and may be made to assume any angle with each other. We will place them so that their dihedral angle is about $90^{\circ}$. Make one of the pieces as nearly as possible horizontal. We will call it the horizontal plane of projection and designate it 
by $H$. The other piece will be approximately vertical in position. We will call it the vertical plane of projection and designate it by $V$. Hold the papers so that $H$ is lower than the eye and $V$ in front of it. The angular space in view we will call the first quadrant and designate it by $1 Q$; the angular space behind $1 Q$ we will call the second quadrant or $2 Q$; that below $2 Q$ will be $3 Q$; that in front of $3 Q$ and below $1 Q$ will be $4 Q$. The medial line of $H$ and $V$, or the hinge, we will call the ground line and designate it by $G$.

Suppose a point situated in space somewhere in $1 Q$, and perpendicular lines drawn from the point to $H$ and $V$ respectively.

Fig. $\beta$.

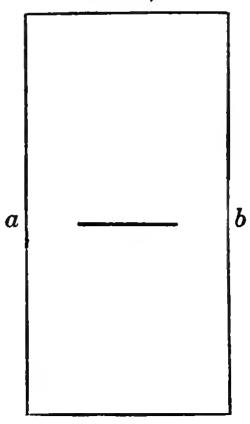

These lines are the projecting lines of the point, and the points in which they pierce $H$ and $V$ are respectively the $H$ and $V$ projections of the point. Assuming our pieces of paper to be true planes at right angles to each other, the two projecting lines determine a third plane perpendicular to both $H$ and $V$, therefore to their intersection $G$. This third plane will cut from $H$ and $V$ two lines each of which is perpendicular to $G$ at the same point. If now we reverse our operation and erect perpendiculars to $H$ and $V$ at the projections of a point, it is evident that they must meet in space and determine the original point. A point is therefore fully determined by its two projections.

When we revolve $H$ and $V$ upon $G$ it is evident that the relation of the intersections of the third plane to $G$ is not changed. When, therefore, $H$ and $V$ form one continuous plane, the two intersections form one and the same right-line perpendicular to $G$. We thus establish the important proposition that when the planes of projection are brought into coincidence the right-line joining the two projections of any point is and must be perpendicular to the ground line.

If instead of a single point in space we had taken a system of points lying in a right-line, the projecting lines of all these 
points would lie in a projecting plane whose intersection with $H$ or $V$ would form the $H$ or $V$ projection of the line. Except in special positions to be studied hereafter the $H$ and $V$ projections of a line determine two projecting planes whose intersection in space is the original line.

Going a step further we see that the locus of all the projecting lines to $H$ or $V$ of all the points of a space curve ${ }^{1}$ is a continuous surface generated by a right-line moving along the curve with all its positions parallel, is a cylindrical surface therefore, and in this case a projecting cylindrical surface whose intersection with its plane of projection is the like-named projection of the space curve. The horizontal and vertical projecting surfaces intersect in space in the original curve. Hence, in general, all the points of any solid in space, therefore the solid itself, are fully determined by their $H$ and $V$ projections.

In order now that this perfect representation of a figure in space may be had upon a single plane surface, we first conceive our planes of projection at right-angles to each other. We then suppose a revolution upon $G$ which brings them into coincidence and perform the operations which this revolution necessitates. $H$ and $V$ are always made coincident by enlarging the angle between upper $V$ and front $H$ from one of $90^{\circ}$ to one of $180^{\circ}$.

As a preliminary exercise let us discuss the first part of problem 3. We will represent, by the method explained above, a line $a b$ crossing the first quadrant. We will also find its true length between $H$ and $V$, and the angles it makes with these planes. Recurring to our two pieces of paper, it is evident that if the line is to cross $1 Q$, the point in which it pierces $V$ must be above $G$, and that in which it pierces $H$ in front of $G$. With these limitations, assume the points of piercing anywhere. Call the point in which $a b$ pierces $H, h$; in which it pierces $V, v . h$ is its own $H$ projection ; the $H$ projection of $v$ is a point in $G$ found by drawing a perpendicular from $v$ to $G$. We thus know two points in the $H$ projection of $a b$, therefore the $H$ projection itself.

Similarly we find the $V$ projection. The former we designate by $a^{\prime} b^{\prime}$, the latter by $a^{\prime \prime} b^{\prime \prime}$, as in Fig. $\gamma$.

1 Part III. Art. 27. 
To find the true length, $L$, of $a b$ between $h$ and $v$, we can use $a^{\prime} b^{\prime}$ from $G$ to $h$ as the leg of a right-angled triangle whose other leg is the perpendicular from $v$ to $G$. The hypothenuse of this triangle is the length sought. A convenient construction is given in Fig. $\gamma$. The basal angle between $a^{\prime} b^{\prime}$ and $a_{11} b_{11}$ is $a^{\prime}$, the inclination of $a b$ to $H$. The same triangle is sometimes more conveniently constructed in $V$. In this case $(a)(b)$, as shown in Fig. $\gamma$, is the hypothenuse and the length along this line from $v$ to $G$ is $L$. This method may be explained by saying that the line $a b$ is revolved about some point in its $H$ projection until it is parallel to $V$ when its $V$ projection is its true magnitude.

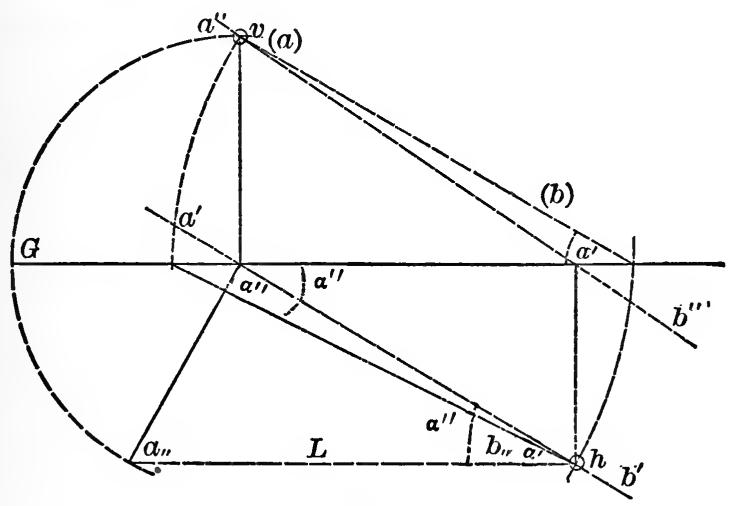

Frg. $\gamma$.

The process here illustrated in $a_{11} b_{11}$ of laying a plane figure over into $H$ or $V$ is called revolution. The relation of the parts of the figure in space to each other is not disturbed, while its $H$ or $V$ projection is taken as the axis of revolution. When some other line in $H$ or $V$ is taken as an axis the figure is said to be developed.

As a rule, points and right-lines are revolved, while points, right-lines, planes, and developable surfaces are developed.

When a plane figure lies in a plane perpendicular to $G$, or in a plane making a very small angle with $G$, a third plane of 
projection is often necessary. When used it is generally taken perpendicular to $G$ and therefore to $H$ and $V$.

To recapitulate briefly.

The planes of projection are three, each one perpendicular to the other two ; one, parallel to the horizon - the first or horizontal plane ; one, perpendicular to this and extending from left to right - the second or vertical plane; one, upon the right extending from front to rear - the third or perpendicular plane, corresponding to what is known in architecture as plan, front and side respectively.

The intersection of the first with the second plane is called the first ground line or simply the ground line; the intersection of the second with the third is taken as the second ground line; the intersection of the third with the first as the third ground line. The first and second planes are revolved about the first ground line into one plane, which therefore represents both; below the ground line is front horizontal and lower vertical; above the ground line is back horizontal and upper vertical. The third plane of projection is revolved, the front part to the right, about the second ground line until it coincides with the second plane.

These transformations are further illustrated in figures 1 and 2,3 and 4 ; figures 1 and 3 show the three planes in space marked $H, V, P$, before revolution and the first, second, third ground lines; figures 2 and 4 show the same lines and planes transformed.

Projection has the same meaning as in elementary Geometry and is effected by projecting lines, planes, and cylindrical surfaces; the intersections of these with the planes of projection are the projections of a figure.

After reading through the remaining portion of the Introduction, the student should proceed to solve the problems with the least possible help from Part II.

The projections of given lines, or the traces of given planes, are represented by an unbroken line: 
Whether the unbroken line belongs to the former or latter is shown in two ways : by the lettering, and by the fact that the traces of a plane always meet on $G$, while in general the projections of a line do not.

When the continuation of a projection of a given line is covered by a plane of projection or by a solid in space, the covered portion is represented by short dashes :

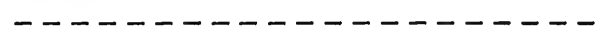

The continuation of the traces of a given plane under similar conditions, by dashes nearly twice as long:

The projections of a required line, or the traces of a required plane, by a line broken at much longer intervals :

while the covered portions of both are represented as in the given line and plane.

The projections of an auxiliary line are alternate dashes and dots :

The traces of auxiliary planes are longer dashes alternating with two dots :

In the last two cases no difference is made in the covered portions, except in a few problems where a distinction seems necessary, when the covered projections and traces are drawn lighter. Given or required arcs or circles are drawn full, auxiliary are broken.

A series of points is used for connecting the projections of points, whether in space or developed.

Developed lines and traces, when neither given nor required, 
are represented as auxiliary ; for, while they are neither new nor in a measure independent figures, they are helps in reaching the result sought.

$H$, means the horizontal plane of projection.

$V$, the vertical plane of projection.

$P$, the perpendicular plane of projection.

$G$, the first ground line.

$G_{2}$, the second ground line.

$G_{3}, \quad$ the third ground line.

$1 Q$, the first quadrant.

$2 Q$, the second quadrant; etc.

gn., given.

rq., required; that is, when not otherwise stated, the first and second projections are required.

pr., projection ; prs., projections.

$H$-pr., the horizontal projection, or the projection in the first plane.

$V$-pr., the second projection.

$P$-pr., the third projection.

$h$, the point in which a line pierces the first plane.

$v$, the point in which a line pierces the second plane.

$p$, the point in which a line pierces the third plane.

$L$, the true length of a line between $h$ and $v$.

line, right-line, unless otherwise qualified, or shown by the context to mean line in general.

pt., point; pts., points.

revolved position, means a position assumed when a space figure is revolved into a plane of projection on a line of this plane through the corresponding projection of the figure as an axis. In Fig. $\gamma, a_{1} b_{1}$ is a rev'd pos. of $a b$. developed position, the same as above, except the axis of revolution is any other line of the corresponding plane of projection; in general, such axis will be the corresponding trace of some oblique plane which contains the figure in space. In Fig. 13, $(a)(b)$ is dev. pos. of $a b$. 
$x, y, z$, ordinates : when a figure is given by its $x, y, z$ ordinates, $x$ means distance from $P$ parallel to $G ; y$, distance from $V$ parallel to $G_{3} ; z$, distance from $H$ parallel to $G_{2}$. Positive $x$ is reckoned from $P$ to the observer's right; positive $y$, from $V$ forwards ; positive $z$, from $H$ upwards. When the position of a figure is given by its $x, y, z$ ordinates the position of $P$ is shifted from the observer's right to his left, to correspond with the usual assumptions of Analytic Geometry.

$a b c$, etc., designates the figure in space.

$a^{\prime} b^{\prime} c^{\prime}$, etc., its first projection.

$a^{\prime \prime} b^{\prime \prime} c^{\prime \prime}$, etc., its second projection.

$a^{\prime \prime \prime} b^{\prime \prime \prime} c^{\prime \prime \prime}$, etc., its third projection.

$a_{1} b_{1} c_{1}$, etc., the figure revolved into $H$.

$a_{2} b_{2} c_{2}$, etc., the figure revolved into $V$.

$a_{3} b_{3} c_{3}$, etc., the figure revolved into $P$. When a figure is revolved into any plane more than once, double subscripts should be used; as, $a_{12}$.

$(a),(b),(c)$, etc., a figure developed in any plane of projection. When several times developed in the same plane of projection the figures may be distinguished by the Arabic numerals; as, $(a)_{1},(a)_{2}$, etc.

$t^{\prime} T t^{\prime \prime}$, or $a b, c d$, a plane in space; the former given by its traces, the latter by two parallel or intersecting lines.

$t^{\prime} T, \quad$ the first trace of the plane $t^{\prime} T t^{\prime \prime}$.

$T t^{\prime \prime}$, the second trace.

$T^{\prime \prime} t^{\prime \prime \prime}$, the third trace.

$K$, the angle which the first and second traces make in space.

$\alpha^{\prime}$, the inclination of a line to $H$, or the minimum acute angle which it makes with any line of $H$ through its foot.

$a^{\prime \prime}$, the inclination of a line to $V$.

$a^{\prime \prime \prime}$, the inclination of a line to $P$.

$\epsilon^{\prime}$, the angle of inclination of a plane to $H$, or the plane angle formed by drawing two lines, one in the plane 
and one in $H$, each perpendicular to the $H$-trace of the plane at the same point.

$\epsilon^{\prime \prime}, \quad$ the angle of inclination of a plane to $V$.

$\epsilon^{\prime \prime \prime}$ the angle of inclination of a plane to $P$.

$\perp$, perpendicular.

II, parallel.

$=$ equal.

$\triangle$, triangle.

$\square$, parallelogram.

$\Varangle, \quad$ a given angle or angle in general.

భ, a required angle.

$>$, greater than.

$<$, less than.

windschief, two lines crossing each other in space but not intersecting.

$\infty$ infinity.

$x, \quad$ given point.

$\odot, \quad$ required point. 


\section{DESCRIPTIVE GEOMETRY.}

\section{PART I.}

\section{EXEROISES AND PROBLEMS.}

In the following exercises and problems the student is referred to the Introduction for explanations of abbreviations and notation ; to Part II. for suggestions, analyses and demonstrations ; and to Part III. for a synopsis of the subject-matter of Descriptive Geometry as covered by this handbook.

\section{Section I.}

Progressive Course on the Point, Line and Plane.

1. Construct the projections of a point, $a$, in each of the four quadrants.

2. Find and construct five other positions of a point, $a$, the first of which shall lie in the fore part of the horizontal plane.

3. Construct four lines : one, $a b$, crossing the first quadrant; one, $c d$, crossing the second quadrant; etc. Find their horizontal and vertical traces, and designate the former by $h$, the latter by $v$.

4. Construct four lines, $a b, c d$, etc., parallel to the ground line, one in each quadrant.

5. Construct a line, $a b$, parallel to the vertical plane, and inclined to the horizontal plane.

6 . Construct a line, $a b$, lying in the horizontal or vertical plane; also a line, $c d$, lying in the bisecting plane of the first and third quadrants, and a line, ef, lying in the bisecting plane of the second and fourth quadrants. 
7. Construct lines, $a b, c d$, etc., crossing the four quadrants and lying in planes perpendicular to the ground line. Use the perpendicular plane in each case as a third plane of projection. Revolve the latter upon its intersection with the vertical plane considered as a second ground line, and show the true position of each third projection, $a^{\prime \prime \prime} b^{\prime \prime \prime}, c^{\prime \prime \prime} d^{\prime \prime \prime}$, etc.

8. Find and represent in a similar way six other positions of lines lying in planes perpendicular to the ground line.

Note. From this point on, the notation and abbreviations explained in the Introduction will be fully used.

9. Construct two lines, $a b, c d$, intersecting in the pt. $a$, one $\|$ to $V$, the other to $H$.

10. Construct any two lines, $a b, c d$, intersecting in the pt. $x, y, z ; x=3, y=2, z=4$. Assume origin in $G$.

11. Construct a cone of revolution,

a) with base, $B$, in $H$ and vertex, $S$, in space;

$b$ ) with $B$ in $V$ and $S$ in $H$.

Show in $a$ ) the inclination, $a^{\prime}$, of elements to $H$; in $b$ ), the inclination, $a^{\prime \prime}$, of elements to $V$.

12. Construct a cylinder of revolution,

a) with $B$ in $H$ and axis in $V$;

b) with $B$ in $V$ and axis $\perp$ to $V$.

13. Gn. a line, $a b$; rq. $a^{\prime}, a^{\prime \prime}$, and true length, $L$, between its traces, $h, v$. Solve at least for lines crossing $1 Q$ and $2 Q$.

14. Gn. two pts. in space, $a$ in $1 Q, b$ in $4 Q$; rq. their true distance.

15. Of a line, $a b$, in space, gn. $a^{\prime} b^{\prime}, h$ and $a^{\prime} ;$ rq. $a^{\prime \prime} b^{\prime \prime}$ and $v$.

16. Of a line, $a b$, gn. $a^{\prime} b^{\prime}, h$ and $a^{\prime \prime}$; rq. $a^{\prime \prime} b^{\prime \prime}, a^{\prime}$ and $v$.

17. Of a line, $a b$, gn. $a^{\prime} b^{\prime}, L$ and $\alpha^{\prime}$; rq. $a^{\prime \prime} b^{\prime \prime}, a^{\prime \prime}, v$ and $h$. Solve at least for $1 Q$ and $2 Q$.

18. Of a line, $a b$, gn. $a^{\prime \prime} b^{\prime \prime}, v$ and $a^{\prime}$; rq. $a^{\prime} b^{\prime}$ and $h$.

19. Of a line, $a b$, gn. $a^{\prime \prime} b^{\prime \prime}, v$ and $a^{\prime \prime}$; rq. $a^{\prime} b^{\prime}$ and $h$.

20. Of a line, $a b$, gn. $h, a^{\prime}$ and $a^{\prime \prime}$; rq. $a^{\prime} b^{\prime}$ and $a^{\prime \prime} b^{\prime \prime}$. Construct all the possible positions of the required line and find the $\Varangle a^{\prime \prime \prime}$, made with the third plane of projection, $P$. 
21. Gn. a line, $a b$; rq. the pt. $p$ of $a b$ equally distant from $H$ and $V$. Solve by $P$.

22. Gn. a plane, $t^{\prime} T t^{\prime \prime}$, and $a^{\prime \prime} b^{\prime \prime}$ of a line therein; rq. $a^{\prime} b^{\prime}$ of the line, $a b$, of the plane.

23. Gn. a plane, $t^{\prime} T t^{\prime \prime}$, and $a^{\prime}$ of a pt., $a$, therein ; rq. $a^{\prime \prime}$.

24. Gn. a plane, $t^{\prime} T t^{\prime \prime}$; rq. its inclinations to $H$ and $V, \epsilon^{\prime}$ and $\epsilon^{\prime \prime}$.

25. Gn. a plane, $t^{\prime} T t^{\prime \prime} ;$ rq. the $\Varangle K$ which the traces of a plane make in space. See Fig. 6 .

26. Of a plane, $t^{\prime} T t^{\prime \prime}$, gn. $t^{\prime} T$ and $K$; rq. $T t^{\prime \prime}$.

27. Of a plane, $t^{\prime} T t^{\prime \prime}$, gn. $K, \epsilon^{\prime}$ and a pt., $p$, in $t^{\prime} T$; rq. $t^{\prime} T$ and $T t^{\prime \prime}$.

28. Of a plane, $t^{\prime} T t^{\prime \prime}$, gn. $t^{\prime} T$ and $\epsilon$; rq. $T t^{\prime \prime}$.

29. Of a plane, $t^{\prime} T t^{\prime \prime}$, gn. $t^{\prime} T$ and $\epsilon^{\prime \prime}$; rq. $T t^{\prime \prime}$.

30. Of a plane, $t^{\prime} T t^{\prime \prime}$, gn. $\epsilon^{\prime}, \epsilon^{\prime \prime}$ and a pt., $p$, in $t^{\prime} T$; rq. $t^{\prime} T$ and $T t^{\prime \prime}$.

31. Gn. a plane, $t^{\prime} T t^{\prime \prime}$, and a pt., $p$, therein ; rq. the developed position, $(p)$, of the pt. when the gn. plane is developed into $H$ on $t^{\prime} T$ or into $V$ on $T t^{\prime \prime}$.

32. Gn. a plane, $t^{\prime} T t^{\prime \prime}$, and the developed position, $(p)$, of a pt., $p$, therein ; rq. $p^{\prime}$ and $p^{\prime \prime}$.

33. Of a pt., $p$, gn. $p^{\prime}, p^{\prime \prime}$ and $(p)$; rq. the plane, $t^{\prime} T t^{\prime \prime}$, containing the pt.

34. Gn. $\epsilon^{\prime \prime}$ of a plane, $t^{\prime} T t^{\prime \prime}$, containing the pt. $p$, and $p^{\prime}$ and $(p)$ of the pt. ; rq. $t^{\prime} T, T t^{\prime \prime}$ and $\epsilon^{\prime}$.

35. Gn. the $\Varangle K$ of a plane, $t^{\prime} T t^{\prime \prime}$, containing the pt. $p$, and $p^{\prime}$ and $(p)$ of the pt.; rq. $t^{\prime} T$ and $T t^{\prime \prime}$.

36. Gn. a plane, $t^{\prime} T t^{\prime \prime}$, and the distances $m^{\prime}$ and $m^{\prime \prime}$; rq. the pt. $p$, whose distances from $t^{\prime} T$ and $T t^{\prime \prime}$ are $m^{\prime}$ and $m^{\prime \prime}$, respectively.

37. Gn. a plane, $t^{\prime} T t^{\prime \prime}$, and a line, $a b$, therein; rq. the developed line, $(a b)$, and the $\Varangle \mathrm{s} \beta^{\prime}$ and $\beta^{\prime \prime}$, which the line $a b$ makes with $t^{\prime} T$ and $T t^{\prime \prime}$, respectively.

38. Gn. two intersecting lines, $a b, c d$; rq. the plane, $t^{\prime} T t^{\prime \prime}$, containing them, and the $\Varangle \delta$ which these lines make in space. Find the bisector, $p q$, of the $\Varangle$ between the gn. lines.

39. Gn. a plane, $t^{\prime} T t^{\prime \prime}$, a line, $a b$, therein, a pt., $p$, in $a b$ and the $\Varangle \delta$; rq. the line $c d$, lying in $t^{\prime} T t^{\prime \prime}$, passing through $p$, and making with $a b$ the $\Varangle \delta$. 
40. Gn. two II lines, $a b, c d$; rq. their plane, $t^{\prime} T t^{\prime \prime}$, and their true distance apart, $D$.

41. Gn. three pts., $a, b, c$; rq. their plane, $t^{\prime} T t^{\prime \prime}$, and their true relation, $(a)(b)(c)$, as shown in $H$ by revolving on $t^{\prime} T$, and in $V$ by revolving on $T t^{\prime \prime}$.

42. Gn. a line, $a b$, and a pt., $p$, without it; rq. the plane, $t^{\prime} T t^{\prime \prime}$, of $a b$ and $p$, also the distance from $p$ to $a b$, found by developing the latter into $V$ upon $T t^{\prime \prime}$.

43. Gn. a line, $a b$, a pt., $p$, without it and the $\Varangle \beta$; rq. the line, $c d$, lying in the plane, $t^{\prime} T t^{\prime \prime}$, which contains $a b$ and $p ; c d$ passes through $p$ and makes with $a b$ the $\Varangle \beta$.

44. Gn. a plane, $t^{\prime} T t^{\prime \prime}$, a pt., $p$, within it and the $\Varangle \delta$; rq. the line $c d$ of $t^{\prime} T t^{\prime \prime}$, containing $p$ and making with $H$ the $\Varangle \delta$.

45. Gn. a line, $a b$, and the $\Varangle \delta$; rq. the plane, $t^{\prime} T t^{\prime \prime}$, containing $a b$ and so situated that $t^{\prime} T$ makes with $a b$ the $\Varangle \delta$.

46. Gn. a plane, $t^{\prime} T t^{\prime \prime}$, and a pt., $p$, without it ; rq. the distance, $D$, from $p$ to $t^{\prime} T t^{\prime \prime}$. Solve by a projecting plane containing $p$ and $\perp$ to $t^{\prime} T$.

47. Of a plane, $t^{\prime} T t^{\prime \prime}$, gn. $t^{\prime} T$, a pt., $p$, without the plane and the distance, $D$, from $p$ to $t^{\prime} T t^{\prime \prime}$; rq. $T t^{\prime \prime}$. Reverse 46 .

48. Gn. a plane, $t^{\prime} T t^{\prime \prime}$, and a line, $a b$, without it ; rq. the pt., $p$, in which $a b$ pierces $t^{\prime} T t^{\prime \prime}$.

1) Line general :

a) Plane general ;

b) Plane $\|$ to $G$.

2) Line in plane $\perp$ to $G$ :
a) Plane general ;
b) Plane $\|$ to $G$.

Solve for each case. In 1) a) make $K$ acute, in 2) a) make $K$ obtuse.

49. Gn. a plane, $t^{\prime} T t^{\prime \prime}$, and a pt., $p$, without it; rq. the distance, $D$, from $p$ to $t^{\prime} T t^{\prime \prime}$. Solve by drawing a $\perp$ from the $p t$. to the plane and determining its foot in the plane and its true length between the pt. $p$ and the foot.

50. Gn. a line, $a b$, and a pt., $p$, without it; rq. the plane, $t^{\prime} T t^{\prime \prime}$, containing $p$ and $\perp$ to $a b$. Also find the distance, $D$, 
from $p$ to $a b$ by finding the length of the line from $p$ to the $p t$. $q$ where $a b$ pierces $t^{\prime} T t^{\prime \prime}$.

51. Gn. a plane, $t^{\prime} T t^{\prime \prime}$, and a line, $a b$, without it; rq. the $\Varangle \delta$ which $a b$ makes with $t^{\prime} T t^{\prime \prime}$. Solve by a $\perp, p h$, from any pt., $p$, of $a b$ upon $t^{\prime} T t^{\prime \prime}$. The $\Varangle$ between $p h$ and $a b=90^{\circ}-\delta$.

52. Gn. a line, $a b$, and a pt., $p$, without it; rq. the line, $p q$, containing $p$ and intersecting $a b$ at rt. angles. This is the extension of 50. In one of the two problems, 50 and 52, assume the data so that $K$ is $>90^{\circ}$, in the other so that $K$ is $<90^{\circ}$.

53. Gn. a plane, $t^{\prime} T t^{\prime \prime}$, a pt., $p$, without it and the $\Varangle \mathrm{s} \delta$ and $\theta$; rq. a line, $p m$, containing $p$, making with $H$ the $\Varangle \delta$ and with $t^{\prime} T t^{\prime \prime}$ the $\Varangle \theta$.

54. Gn. two intersecting planes, $t^{\prime} T t^{\prime \prime}$ and $r^{\prime} R r^{\prime \prime}$; rq. their line of intersection, $m n$,

a) when the traces intersect within the limits of the drawing ;

b) when the traces do not so intersect.

55. Gn. two intersecting planes, $t^{\prime} T t^{\prime \prime}$ and $r^{\prime} R r^{\prime \prime}$; rq. the $\Varangle \phi$ between them. Bisect the $\Varangle \phi$ by a third plane, $s^{\prime} S s^{\prime \prime}$.

56. Gn. two $\|$ planes, $t^{\prime} T t^{\prime \prime}$ and $r^{\prime} R r^{\prime \prime}$; rq. their distance apart, $D$.

57. Gn. two windschief lines, $a b$ and $c d$; rq. the plane, $t^{\prime} T t^{\prime \prime}$, containing $a b$ and $\|$ to $c d$.

58. Gn. two windschief lines, $a b$ and $c d$, and the pt. $p$; rq. the plane, $t^{\prime} T t^{\prime \prime}$, containing $p$ and $\|$ to $a b$ and $c d$.

59. Gn. two windschief lines, $a b$ and $c d$; rq. their distance, $D$, and its projections, $p^{\prime} q^{\prime}$ and $p^{\prime \prime} q^{\prime \prime}$.

60. Gn. two windschief lines, $a b$ and $c d$, and the pt. $p$; rq. the line, $p q$, passing through $p$ and cutting both $a b$ and $c d$. Solve, a) with the traces of auxiliary planes;

b) without such traces.

61. Gn. a plane, $t^{\prime} T t^{\prime \prime}$, and a pt., $p$, without it; rq. a plane, $r^{\prime} R r^{\prime \prime}$, containing $p$ and $\|$ to $t^{\prime} T t^{\prime \prime}$.

62. Gn. a plane, $t^{\prime} T t^{\prime \prime}$, and the distance $D$; rq. a plane, $r^{\prime} R r^{\prime \prime}, \|$ to $t^{\prime} T t^{\prime \prime}$ and at the distance $D$. 
63. Gn. a plane, $t^{\prime} T t^{\prime \prime}$, and without it a line, $a b$, and a pt., $p$; rq. a plane, $r^{\prime} R r^{\prime \prime}$, containing $p$, $\|$ to $a b$ and $\perp$ to $t^{\prime} T t^{\prime \prime}$.

64. Gn. a plane, $t^{\prime} T t^{\prime \prime}$, a line, $a b$, within it and the $\Varangle \delta$; rq. a plane, $r^{\prime} R r^{\prime \prime}$, containing $a b$ and making with $t^{\prime} T t^{\prime \prime}$ the $\Varangle \delta$. Construct when $\delta=60^{\circ}$.

65. Gn. a plane, $t^{\prime} T t^{\prime \prime}$, a line, $a b$, without it and the $\Varangle \phi$; rq. a plane, $r^{\prime} R r^{\prime \prime}$, containing $a b$ and making with $t^{\prime} T t^{\prime \prime}$ the $\Varangle \phi$.

66. Gn. a plane, $t^{\prime} T t^{\prime \prime}$, a line, $a b$, within it, a pt., $p$, without it and the $\Varangle \delta$; rq. a line, $p q$, connecting $p$ and $a b$ and making with $t^{\prime} T t^{\prime \prime}$ the $\Varangle \delta$.

Note. In trihedrals the face $\Varangle \mathrm{s}$ are $a, \beta, \gamma$, and the opposite dihedrals are $A, B, C$, respectively.

67. Gn. $\alpha, \beta, \gamma ;$ rq. $A, B, C$.

68. Gn. $\alpha, \beta, C$; rq. $A, B, \gamma$.

69. Gn. $\alpha, \beta, A$; rq. $B, C, \gamma$.

70. Gn. $\beta, C, A$; rq. $B, \gamma, \alpha$.

71. Gn. $\alpha, C, A$; rq. $B, \beta, \gamma$.

72. Gn. $A, B, C$; rq. $\alpha, \beta, \gamma$.

73. Gn. $\alpha, C,(\beta+\gamma)$; rq. $A, B, \beta, \gamma$.

74. Gn. $\alpha, C,(\beta-\gamma)$; rq. $A, B, \beta, \gamma$.

75. Gn. a plane, $t^{\prime} T t^{\prime \prime}$, and $a^{\prime} b^{\prime} c^{\prime} d^{\prime} e^{\prime}$ of a pentagon, abcde, lying therein; rq. $a^{\prime \prime} b^{\prime \prime} c^{\prime \prime} d^{\prime \prime} e^{\prime \prime}$ and the true figure, $(a)(b)(c)$ (d) $(e)$.

76. Of a plane pentagon, abcde, gn. $a^{\prime} b^{\prime} c^{\prime} d^{\prime} e^{\prime}$ and $a^{\prime \prime} b^{\prime \prime} c^{\prime \prime}$; rq. $d^{\prime \prime} e^{\prime \prime}$ and the true figure, $(a)(b)(c)(d)(e)$, without constructing the plane of the pentagon.

77. Of a plane rectangle, $a b c d$, gn. $a^{\prime} b^{\prime} c^{\prime} d^{\prime}$ and the true length, $L$, of the side $a b$; one vertex, $a$, lies in $H$; rq. $a^{\prime \prime} b^{\prime \prime} c^{\prime \prime} d^{\prime \prime}$, the plane, $t^{\prime} T t^{\prime \prime}$, of the rectangle and the true figure, $(a)(b)$ (c) $(d)$.

78. Gn. a pyramid, $S-a b c$, and $p^{\prime \prime}$ of a pt., $p$, upon its surface; rq. $p^{\prime}$. Assume the pyramid with its base in $H$.

79. Gn. a pyramid, $S$-abcd; rq. the length, $L$, of the edge $S a$, the $\Varangle a^{\prime \prime}$ of inclination of $S a$ to $V$, the $\Varangle \phi$ between the two faces $S a b$ and $S b c$, the $\Varangle \epsilon^{\prime \prime}$ of inclination of the face $S c d$ 
to $V$ and the development of the pyramid in $V,(S)(a)(b)$ (c) $(d)$, the solid being opened on the edge $S c$ and the developed lateral surface remaining attached in $V$ to the basal edge $a b$. Assume the pyramid with the base in $V$.

80. Of a triangular pyramid, $S$ - $a b c$, gn. its base, $a b c$, in $H$ and the lengths $L_{1}, L_{2}, L_{3}$, of the edges $S a, S b, S c$, respectively ; rq. the projections of the pyramid.

81. Of a triangular pyramid, $S-a b c$, gn. its base, $a b c$, in $V$, two lateral edges, $S a, S b$, and its altitude, $A$; rq. the projecjections of the pyramid.

82. Of a triangular pyramid, $S$ - $a b c$, gn. the face $\Varangle \mathrm{s} \alpha, \beta, \gamma$, of the trihedral at the vertex $S$, one basal edge, $a b$, and the conditions that the base shall lie in $H$ and that the inclinations of the lateral faces to $H$ shall be equal ; rq. the projections of the pyramid.

83. Of a triangular pyramid, $S$-abc, gn. the face $\Varangle \mathrm{s} a, \beta, \gamma$, at the vertex $S$, two lateral edges, $S a, S b$, and the $\nvdash \epsilon^{\prime}$ of inclination,

a) of the face $S a b$ to $H$;

b) of the face $S a c$ to $H$;

rq. the projections of the pyramid. Assume the base in $H$.

84. Gn. a triangular pyramid, $S \bullet a b c$; rq. the circumscribed sphere and its centre, $C$.

85. Gn. a triangular pyramid, $S-a b c$; rq. the inscribed sphere and its centre, $C$.

86. Gn. an edge, $a b$, in $H$ of

a) a regular tetrahedron;

b) a regular octahedron;

c) a regular icosahedron;

d) a regular dodecahedron ;

rq. the projections of the polyhedrons and their development in $H$. $a b$ is to be assumed in each case as one side of a face lying in $H$, upon which the polyhedron rests. No one of the sides of this basal face is to be taken either $\|$ or $\perp$ to $G$.

87. Gn. an edge, $a b$, of a regular icosahedron $\|$ to $H$ and an 
adjacent edge, ac, making $\Varangle \mathrm{s}$ of $30^{\circ}$ and $45^{\circ}$ with $H$ and $V$ respectively; rq. the projections of the solid.

88. Gn. a plane, $t^{\prime} T l^{\prime \prime}$, and a pyramid, $S$ - $a b c$; rq. to determine whether the plane cuts the pyramid between the limits of the vertex and base.

89. Gn. a pyramid, $S$ - $a b c$, and an intersecting plane, $t^{\prime} T t^{\prime \prime}$; rq. the figure of intersection, mno.

90. Gn. a prism, $a b c-a_{1} b_{1} c_{1}$, and an intersecting plane, $t^{\prime} T t^{\prime \prime}$; rq. the figure of intersection, mno.

91. Of the hexagonal pyramid, $S$-abcdef, gn. the base, abcdef, in $H$ and the lengths $L_{1}, L_{2}, L_{3}$, of $S a, S c, S e$, respectively; rq. the pyramid and its intersection, mno etc., by a plane, $t^{\prime} T t^{\prime \prime}$, which cuts off equal distances from $S a, S c, S e$.

92. Gn. a line, de, and a pyramid, $S$-abc; rq. the pts. $m, n$, in which de pierces $S$ - $a b c$.

93. Gn. a line, de, and a prism, $a b c-a_{1} b_{1} c_{1}$; rq. the pts. $m, n$, of intersection.

94. Gn. two intersecting pyramids, $S$-abcd and $T-x y z$, with bases in $H$; rq. the figure of intersection, mno etc.

95. Gn. two intersecting prisms, $a b c d-a_{1} b_{1} c_{1} d_{1}$ and $x y z-x_{1} y_{1} z_{1}$, with bases in $V$; rq. the figure of intersection, mno etc.

96. Gn. a prism, $a b c d-a_{1} b_{1} c_{1} d_{1}$, with base in $H$ and an intersecting pyramid, $S-x y z$, with base in $V$; rq. the figure of intersection, mno etc.

97. Gn. unit rectangular axes, ox, oy, oz, $\|$ to the intersections of $H V, H P, V P$, respectively ; rq. their projections, $o_{1} x_{1}$, $o_{1} y_{1}, o_{1} z_{1}$, upon a fourth plane, $t^{\prime} T t^{\prime \prime}$, which is inclined to $H$ at an $\Varangle$ of $80^{\circ}$ and whose $t^{\prime} T$ makes with $G$ the $\Varangle 30^{\circ}$, also rq. the development of $t^{\prime} T t^{\prime \prime}$ on $t^{\prime} T$ in $H$ and the projected axes $\left(o_{1}\right)\left(x_{1}\right),\left(o_{1}\right)\left(y_{1}\right),\left(o_{1}\right)\left(z_{1}\right)$.

98. Project axonometrically the star-dodecahedron, the ratio of unit rectangular axes in space being in projection, $x: y: z$ $=\frac{9}{10}: \frac{1}{2}: 1$; the inclinations of the projected axes to $G$ being $\tan \Varangle x o G=\frac{1}{11}, \tan \Varangle y o G=\frac{1}{3}, \tan \Varangle z o G=\infty$.

99. Gn. in $H$ two $\AA$, $a b c$ and $m n o$; rq. the projections of $a b c$ when the $H$ projection, $a^{\prime} b^{\prime} c^{\prime}$, is similar to mno. Let the 
vertex $c$ remain in $H$ during the necessary rotation of the plane of $a b c$.

100. Gn. the $H$ projection, $a^{\prime} b^{\prime} c^{\prime}$, of a $\triangle a b c$, the altitude of the vertex $b$ and another $\triangle, m n o$; rq. the $V$ projection, $a^{\prime \prime} b^{\prime \prime} c^{\prime \prime}$, when $a b c$ is similar to mno.

101. Of the three concurrent edges of a cube, $O A, O B, O C$, the $V$ projections, $O^{\prime \prime} A^{\prime \prime}, O^{\prime \prime} B^{\prime \prime}$, of two are known ; rq. the corresponding projection, $O^{\prime \prime} C^{\prime \prime}$, of the third.

102. Gn. $O^{\prime \prime} A^{\prime \prime}$ as in 101 and the directions of $O^{\prime \prime} B^{\prime \prime}$ and $O^{\prime \prime} C^{\prime \prime}$; rq. the corresponding lengths of the two last.

103. Gn. $O^{\prime \prime} A^{\prime \prime}$ as in 101 and the lengths, $L_{1}, L_{2}$, of $O^{\prime \prime} B^{\prime \prime}$, $O^{\prime \prime} C^{\prime \prime}$; rq. the corresponding positions of the two last.

104. Gn. $O^{\prime \prime} A^{\prime \prime}$ as in 101 , the direction of $O^{\prime \prime} B^{\prime \prime}$ and the length, $L_{2}$, of $O^{\prime \prime} C^{\prime \prime}$; rq. the corresponding length of $O^{\prime \prime} B^{\prime \prime}$ and position of $O^{\prime \prime} C^{\prime \prime}$.

\section{SeCtion II.}

Additional Exercises upon the Point, Line and Plane.

105. Gn. three pts., $a, b, c$, as the vertices of a triangle, $a b c$, by $a^{\prime}, b^{\prime}, c^{\prime}$, and the distances $d_{1}, d_{2}, d_{3}$, respectively above $H$; rq. $a^{\prime \prime} b^{\prime \prime} c^{\prime \prime}$ and the true figure of the $\triangle a b c$.

106. In a plane, $t^{\prime} T t^{\prime \prime}$, given by $t^{\prime} T$ and a pt. $a$, lies a regular octagon with $a$ for its centre and one side, $b c$, $\|$ to $t^{\prime} T$; rq. the prs. of the octagon.

107. Gn. in $H$ a $\square, a b c d$, and the distance $d$; rq. the prs. of $a b c d$ when its centre, $m$, is at the distance $d$ above $H$ and $a^{\prime} b^{\prime} c^{\prime} d^{\prime}$ is a square.

108. Gn. the $H$-pr., $a^{\prime} b^{\prime} c^{\prime}$, of a $\Delta$; rq. the $V$-pr., $a^{\prime \prime} b^{\prime \prime} c^{\prime \prime}$, when $a b c$ in space is equilateral and the vertex, $a$, lies in $H$.

109. Gn. a pt., $o$, as centre of a regular hexagon and the $H$-pr., $a^{\prime} b^{\prime}$, of one side, $a b$; rq. the hexagon, abcdef, and its plane, $t^{\prime} T t^{\prime \prime}$.

110. Gn. the side, $a b$, of a square, $a b c d$, and the $\Varangle \delta$; rq. the square and its plane, $t^{\prime} T t^{\prime \prime}$, when the diagonal ac makes with $H$ the $\Varangle \delta$. 
111. Gn. two pts., $a, b$, and the distance $d$; rq. the equilateral $\triangle a b c$ when $c$ is at the distance $d$ from $H$.

112. Of a $\Delta, a b c$, there are given the vertices $a, b$, the $H$-pr. $c^{\prime}$, a square, $K$, of area equal to that of $a b c$; rq. $c^{\prime \prime}$, the plane, $t^{\prime} T t^{\prime \prime}$, of the $\triangle a b c$ and the true figure, $(a)(b)(c)$, of the latter.

113. Gn. a pt., $p$, a line, $a b, \perp$ to $H$ and a line, $c d, \perp$ to $V$; rq. the line, $p q$, through $p$ and intersecting $a b$ and $c d$.

114. Gn. three lines, $a b, c d$, ef; rq. $\| \mathrm{s}, a_{1} b_{1}, c_{1} d_{1}, e_{1} f_{1}$, to the three lines respectively, each of which shall cut the two given lines to which it is non-parallel.

115. Five pts., $a, b, c, d, e$, are gn. by their co-ordinates, as follows :

\begin{tabular}{rcrcr}
$x$ & $b$ & $c$ & $d$ & \multicolumn{1}{c}{$r$} \\
$x=0$ & 30 & 48 & 40 & 10 \\
$y=15$ & 35 & 6 & 46 & 28 \\
$z=48$ & 10 & 30 & 48 & 0
\end{tabular}

rq. the true figure, $(a)(b)(c)$, of the $\triangle a b c$ and its plane, $t^{\prime} T t^{\prime \prime}$, the distances, $d_{1}, d_{2}$, of $d$ and $e$ from $t^{\prime} T t^{\prime \prime}$ and the pt. $q$ in which the line de pierces $t^{\prime} T t^{\prime \prime}$.

116. Gn. a pt., $p$, and a line, $a b$; rq. the regular decagon, $m n o$ etc., whose centre is $p$ and one of whose sides, $m n$, falls in $a b$.

117. Gn. a plane, $t^{\prime} T t^{\prime \prime}$, and figure, $a b c$ etc., therein, whose $H$-pr., $a^{\prime} b^{\prime} c^{\prime}$ etc., is a regular octagon; rq. $a^{\prime \prime} b^{\prime \prime} c^{\prime \prime}$.

118. Gn. a pt., $p$, and two lines, $a b$ and $c d$, both $\perp$ to $G$; rq. the line, $p q$, through $p$ and intersecting $a b$ and $c d$.

119. Given a plane, $t^{\prime} T t^{\prime \prime}$, and a pt., $p$, without it ; rq. a line, $p q$, through $p$ and $\|$ to $t^{\prime} T t^{\prime \prime}$, whose prs., $p^{\prime} q^{\prime}$ and $p^{\prime \prime} q^{\prime \prime}$, shall be $\|$ lines, when $V$ and $H$ are made to coincide as usual.

120. Gn. a line, $a b$, and two planes, $t^{\prime} T t^{\prime \prime} \perp$ to $H$ and $r^{\prime} R r^{\prime \prime}$ $\perp$ to $V$; rq. the pt., $p$, of $a b$ equally distant from $t^{\prime} T t^{\prime \prime}$ and $r^{\prime} R r^{\prime \prime}$.

121. Gn. a pt., $p$, and the planes, $t^{\prime} T t^{\prime \prime}$ and $r^{\prime} R r^{\prime \prime}$; rq. the line, $p q$, through $p \|$ to both planes.

122. Gn. a line, $a b$, and a plane, $t^{\prime} T t^{\prime \prime}$; rq. the line, $p q$, of $t^{\prime} T t^{\prime \prime} \perp$ to $a b$. 
123. Gn. a pt., $p$, and two windschief lines, $a b$ and $c d$; rq. the line, $p q$, passing through $p$, cutting $a b$ and $\perp$ to $c d$; or, rq. the line, $p t$, passing through $p$ and $\perp$ to both $a b$ and $c d$.

124. Gn. a plane, $t^{\prime} T t^{\prime \prime}$, and two lines, $a b$ and $c d$; rq. the line, $p q, \perp$ to $t^{\prime} T t^{\prime \prime}$ and intersecting $a b$ and $c d$.

125. Gn. a line, $a b$, and the $\Varangle \delta$; rq. the line, $p q$, of $H$, which makes with $a b$ the $\Varangle \delta$.

126. Gn. a pt., $p$, a line, $a b$, and the $\Varangle 30^{\circ}$; rq. the line, $p q$, passing through $p$, cutting $a b$ and making with $V$ the $\Varangle 30^{\circ}$.

127. Gn. a plane, $t^{\prime} T t^{\prime \prime}$, the lines $a b$ and $c d$, and the distance $d$; rq. the line, $p q, \|$ to $t^{\prime} T t^{\prime \prime}$ at the distance $d$ and intersecting $a b$ and $c d$.

128. Gn. two planes, $t^{\prime} T t^{\prime \prime}$ and $r^{\prime} R r^{\prime \prime}$, a line, $a b$, and ratio $m, m_{1}$; rq. the pt., $p$, of $a b$ at the distances $q, q_{1}$, from $t^{\prime} T t^{\prime \prime}$ and $r^{\prime} R r^{\prime \prime}$ respectively: gn. $q: q_{1}=m: m_{1}$.

129. Gn. three planes, $t^{\prime} T t^{\prime \prime}, r^{\prime} R r^{\prime \prime}, s^{\prime} S s^{\prime \prime}$, and the distances $m, n, q$; rq. the pt., $p$, at the distances $m, n, q$, from the three planes respectively.

130. Gn. two pts., $a, b$, the plane $t^{\prime} T t^{\prime \prime}$ and the lengths $L_{1}$, $L_{2}$; rq. the $\triangle, a b c$, constructed upon $a b$ as a base, with sides $a c, b c$, respectively equal to $L_{1}, L_{2}$, and with the vertex $c$ in $t^{\prime} T t^{\prime \prime}$.

131. Gn. two pts., $a, b$, a line, $m n$, and an area, $A$; rq. a pt., $c$, in $m n$, so situated that the $\triangle a b c$ shall have the area $A$.

132. Gn. a line, $a b$, a pt., $p$, and the distance $d$; rq. the plane, $t^{\prime} T t^{\prime \prime}$, containing $a b$ and at the distance $d$ from $p$.

133. Gn. three pts., $a, b, c$, no one of which lies in $H$ or $V$; rq. a pt., $p$, in $H$, equally distant from $a, b, c$.

134. Gn. two pts., $p, q$, the line $a b$ and the distance $d$; rq. the line, $q r$, through $q$, intersecting $a b$ and at the distance $d$ from $p$.

135. Given two distances, $D_{1}, D_{2}$, and

a) three pts., $p, q, r$,

b) two pts., $p, q$, and a line, $a b$,

c) one pt., $p$, and two lines, $a b, c d$; 
rq. a) a line, pt, through $p$ at the distances $D_{1}, D_{2}$, from $q, r$, respectively,

b) a line, $p t$, through $p$, at the distance $D_{1}$ from $q$, and $D_{2}$ from $a b$,

c) a line, $p t$, through $p$, at the distances $D_{1}, D_{2}$, from $a b, c d$, respectively.

136. Gn. four pts., $a, b, c, d$, not in the same plane, and the distances $m, n, p, q$; rq. a fifth pt., $x$, whose distances from $a, b, c, d$, shall be as $m: n: p: q$, respectively.

Take $a, b, c$, in $H$. How many solutions?

137. Gn. four pts., $a, b, c, d$, not in the same plane and the distances $m, n, p, q$; rq. a plane, $t^{\prime} T l^{\prime \prime}$, whose distances from $a, b, c, d$, shall be as $m: n: p: q$, respectively.

Take $a, b, c$, in $H$. How many solutions ?

138. Gn. three pts., $a, b, c$, and the distances $m, n, p$; rq. a plane, $t^{\prime} T l^{\prime \prime}$, whose distances from $a, b, c$, shall be $m, n, p$, respectively.

How many solutions?

139. Gn. four pts., $a, b, c, d$, not lying in the same plane and the quantities $m, n, p, q$; rq. a fifth pt., $x$, whose distances from the planes $a b c, a b d, a c d, b c d$, shall be
a) as $m: n: p: q$,
b) equal.

140. Gn. a pt., $p$, two windschief lines, $a b, c d$, and the $\Varangle \mathrm{s}$ $\delta$ and $\theta$; rq. a line, $p q$, passing through $p$ and making with $a b$, $c d$, the $\Varangle \mathrm{s} \delta, \theta$, respectively.

141. Gn. a pt., $p$, two planes, $t^{\prime} T t^{\prime \prime}, r^{\prime} R r^{\prime \prime}$, and the $\Varangle \mathrm{s} \delta^{\prime}, \delta^{\prime \prime}$; rq. the plane, $x^{\prime} X x^{\prime \prime}$, making with $t^{\prime} T t^{\prime \prime}, r^{\prime} R r^{\prime \prime}$, the $\Varangle \mathrm{s} \delta^{\prime}, \delta^{\prime \prime}$, respectively.

142. Gn. two windschief lines, $a b, c d$, and the $\Varangle \mathrm{s} \beta^{\prime}, \beta^{\prime \prime}$; rq. a line, $p q$, intersecting $a b, c d$, and making with them the $\Varangle \mathrm{s} \beta^{\prime}$, $\beta^{\prime \prime}$, respectively.

143. Gn. a line, $a b$, and two pts., $m, n$; rq. in $a b$ the pt., $p$, the sum or difference of whose distances from $m$ and $n$ shall be a minimum or a maximum respectively.

144. Gn. a plane, $t^{\prime} T t^{\prime \prime}$, and two windschief lines, $a b, c d$; rq. 
the minimum line, $p q$, which is $\|$ to $t^{\prime} T t^{\prime \prime}$ and cuts $a b$ and $c d$.

145. Gn. a plane figure, $a b c$, etc., of $n$ sides, whose area is $A$; let the areas of its projections upon $H, V, P$, be $A_{1}, A_{2}, A_{3}$, respectively; rq. to prove that $A^{2}=A_{1}{ }^{2}+A_{2}{ }^{2}+A_{3}{ }^{2}$.

146. Of a trihedral angle, gn. $a) \alpha, \beta,(A+B+C)$, or $b) A$, $B,(\alpha+\beta+\gamma)$; rq. a) $\gamma, A, B, C, b) C, \alpha, \beta, \gamma$.

147. Of a trihedral angle, gn. $a$, the $\Varangle \epsilon_{1}$ of elevation opposite, and the $\Varangle \epsilon_{2}$ of elevation adjacent to the face of $\alpha ;$ rq. $\beta, \gamma$, and the $\Varangle \epsilon_{3}$ of elevation.

148. Of a trihedral angle, gn. $\alpha$ and the adjacent $\zeta \mathrm{s}$ of elevation $\epsilon_{2}, \epsilon_{3} ; \mathrm{rq} . \beta, \gamma, \epsilon_{1}$.

149. Of a quadrahedral $\Varangle, S$, gn. all the face $\Varangle \mathrm{s}, \alpha, \beta, \gamma, \delta$, and the dihedral $\Varangle, A$, lying between $\alpha$ and $\beta$; rq. the complete projection of the quadrahedral $\Varangle S$.

150. Of a quadrahedral $\Varangle . S$, gn. $\alpha, \beta, \gamma$, and the dihedrals $C, D$, both adjacent to the unknown face $\Varangle \delta$; rq. the complete projection of the quadrahedral $S$.

151. Of a regular tetrahedron, $a b c d$, gn. the vertices $a, b$, and the $\Varangle 45^{\circ}$ as the inclination which the line $c d$, joining $c$ and $d$, makes with $H$; rq. the tetrahedron.

152. Given three concurrent edges, $a b, a c, a d$, of a parallelopiped and the face $\Varangle \mathrm{s}, \alpha, \beta, \gamma$, between $a b, a c ; a c, a d ; a d$, $a b$, respectively; rq. the parallelopiped $a b c d e f g h$.

153. Gn. two pts., $a, h$, as the opposite vertices of a cube, abcdefgh, and the $\Varangle 30^{\circ}$ as the inclination of the edge $a b$ to $H$; rq. the cube.

154. Gn. a cube, abcdefgh; rq. the solid mno etc., formel by passing through the edges of the cube planes each $\perp$ to the diagonal plane in which the edge lies.

155. Gn. in $H$ a $\triangle, a^{\prime} b^{\prime} c^{\prime}$, as the $H$-pr. of a face of a regular tetrahedron, $a b c d$, and $d$, the distance above $H$ of its centre, $o$; rq. the tetrahedron.

156. Gn. two pts., $a, b$, as the terminations of an erge of a regular icosahedron, and the $\Varangle_{\epsilon} ;$ rq. the icosahedron when one of its faces, $a b c$, makes with $H$ the $\Varangle \epsilon$ 。 
157. Gn. three pts., $b, c, d$, as the terminations of three concurrent edges, $a b, a c, a d$, of a rectangular parallelopiped; rq. the projections of the parallelopiped abcdefgh.

158. Of a hexagonal pyramid, $S$-abcdef, gn. the regular hexagonal base, abclef, the radius, $R$, of the circumscribed sphere, the altitude, $A$, of the pyramid and the $\Varangle \beta$ which the side $S a$ makes with $H$; a plane, $t^{\prime} T t^{\prime \prime}$, passes through the centre $o$, of the sphere, cutting from the edges $S a, S c, S e$, the equal lengths $S m, S n, S q$; rq. the projections of the truncated pyramid abcdef-mxnyqz.

159. Of a quadrilateral pyramid, $S$-abcd, gn. the base, abcd, in $H$, the $\Varangle \in$ which the face $S a b$ makes with $H$, the $\Varangle \mathrm{s} \delta_{1}$, $\delta_{2}$, which the face $S a b$ makes with the faces $S a c$, $S b d$, respectively, and the area $A$; rq. the pyramid $S$-abcd and a plane section, mnop, in the form of a parallelogram and of the area $A$.

160. Of a pentagonal truncated prism, $a b c d e-a_{1} b_{1} c_{1} d_{1} e_{1}$, there is given in $H$ the base $a b c d e$, the face $a b a_{1} b_{1}$ and the $\Varangle \beta$ which the edges make with $H$; rq. the prism.

161. Of a tetrahedron, $a b c d$, gn. the edges $a b, a c, a d, b c, b d$, and the radius, $R$, of the circumscriber sphere, also an area, $A$; rq. the tetrahedron, also a section, $m n p q$, in the form of a parallelogram and of the area $A$.

162. Of a regular pentagonal pyramid, $S$-abcde, gn. the altitude, $A$, the length, $L$, of the basal edge $a b$, the plane of the base, $t^{\prime} T t^{\prime \prime}$, which makes with $H$ the $\Varangle 30^{\circ}$, and whose $H$ trace, $t^{\prime} T$, makes with $G$ the $\Varangle 45^{\circ}, a b$ lies in $t^{\prime} T$; rq. the prism.

163. Of a hexagonal pyramid, $S$-abcdef, gn. the base abcdef in $H$, the length, $L$, of the edge $S \alpha$ and the $\Varangle \mathrm{s} \alpha, \beta$, which the edges $S c$, $S e$, respectively make with $H$; rq. the pyramid.

164. Of a quadrilateral pyramid, $S$-abcd, with base in $H$, gn. the faces $S a b, S a d$, the $\Varangle \delta$ between them and the $\Varangle_{\mathrm{s}} \epsilon_{1}$, $\epsilon_{2}$, which the faces $S b c, S c d$, make respectively with $H$; rq. the pyramid and a section, mno, made by a plane, $t^{\prime} T t^{\prime \prime}$, equally inclined to $S a b, S a d$ and $H$. 
165. Gn. a pt., $\alpha$, as a vertex of a cube, $a b c d e f g h$, the length, $L$, of an edge of the cube and a $\Delta, m n o$, in $H$; rq. the prs. of the cube when the sides of the $H$-pr. are $\|$ to $m n o$.

166. Gn. a pt., $S$, passing through $S$, the lines $S m, S n, S p$, and the lengths $L_{1}, L_{2}$; rq. a pyramid, $S$-abcd, with $S$ for its vertex, with three lateral edges, $S a, S b, S c$, lying in $S m, S n, S p$, respectively, with the lateral edges $S a, S b$, equal to $L_{1}, L_{2}$, respectively, and with the base a rectangle, abcd.

167. Gn. a regular dodecahedron and an intersecting plane, $t^{\prime} T t^{\prime \prime}$; rq. the figure of intersection, mno etc.

168. Gn. an octahedron, abcdef, and an intersecting tetrahedron, ghik; rq. the figure of intersection, mno etc.

169. Gn. a regular dodecahedron and a concentric intersecting cube ; rq. the figure of intersection, mno etc.

170. Gn. a cube; rq. the rhombic dodecahedron formed by truncating each edge with a plane passing throngh the centres of the four adjacent edges. Project the new solid, axonometrically, as given in 98 .

\section{Section III.}

Progressive Course on Lines and Surfaces of an Order higher than the first.

171. Given a parallelogram, $a b c d$; rq. by pts.,

a) the inscribed ellipse,

$b)$ the hyperbola with the diagonals of the $\square$ as asymptotes and tangent to two opposite sides. Construct the principal axes.

c) the parabola, tangent to $a b$ at its middle pt., o, and passing through the pts., $c d$. Find the principal axis, ox, and the tangent at its vertex, oy.

Make three different $[\mathrm{s}$ for $a), b), c$ ).

172. a) Construct the ellipse as the envelope of tangents drawn in a gn. $\square$, abcd.

b) Construct the hyperbola as the envelope of tangents drawn between two gn. intersecting lines, $a b, c d$, used as asymptotes. 
c) Construct the parabola as the envelope of tangents drawn between two gn. intersecting lines, $a b, c d$.

173. Gn. the principal axes, $A A^{\prime}, B B^{\prime}$, of an ellipse, or any two conjugate diameters, $a a^{\prime}, \beta \beta^{\prime}$; rq. the ellipse by circles about the principal axes or conjugate diameters.

174. Gn. any two conjugate diameters, $\alpha \alpha^{\prime}, \beta \beta^{\prime}$, of an ellipse ; rq. its principal axes.

175. Gn. the convex plane pentagon abcde; rq. the ellipse determined

a) by the five vertices as pts.,

b) by the five sides as tangents.

Find the principal axes, $A A^{\prime}, B B^{\prime}$, of the ellipse.

176. Gn. the plane pentagon $a b c d e$, in which the pt. $e$ falls within the convex quadrilateral described with $a, b, c, d$, as vertices ; rq. the hyperbola determined

a) by the fire pts. as vertices,

$b$ ) by the five sides as tangents; the intersections of any sixth tangent, $p q$, with $a b$ and $a c$, are to be determined.

177. Gn. a $\triangle, a b c$, and a direction, $m n$, in its plane; rq. the parabola with axis $\|$ to $m n$, passing through $a, b, c$; also the principal axis, ox, and the tangent at its vertex, oy.

178. Gn. any four pts., $a, b, c, d$, in a plane and a tangent line, $p q$, passing through $a$; rq. the conic section thereby determined.

179. Gn. an oblique cylindrical surface with circular base in $H$ and the $V$-pr., $a^{\prime \prime}$, of the pt. $a$ upon it; rq. $a^{\prime}$.

180. Gn. an oblique conical surface with circular base in $V$ and the $I$-pr., $a^{\prime}$, of the pt. $a$ upon it; rq. $a^{\prime \prime}$.

181. Of a cylinder of revolution, $a b c-a_{1} b_{1} c_{1}$, gn. the axis, $o o$, and the radius, $R$, of the base, $a b c$; rq. the cylinder.

182. Of a cone of revolution, $S$-abc, gn. an element, $S a$, upon which it rests in $H$ and the radius, $R$, of the base $a b c$; rq. the cone.

183. Gn. an oblique cone, $S$-abc, with circular base in $H$ and a pt., $p$, on its surface; rq. the development, $(S)-(a)(b)$ $(c)$, of the surface and $(p)$ of the pt. 
184. Gn. an oblique half-cylinder, $a b c-a_{1} b_{1} c_{1}$, resting upon $H$ with concave side downwards and having circles as the $V$-prs. of its bases, also a pt., $p$, thereon ; rq. the development $(a)(b)-\left(a_{1}\right)\left(b_{1}\right)$ in $H$ of the cylinder and $(p)$ of the pt.

185. Gn. cones of rerolution $S$-abc, with bases in $H$ and planes, $t^{\prime} T t^{\prime \prime}$, intersecting them at various angles with the bases $a b c$; rq. sections, mno etc., and their development in $H,(m)$ (n) (o) etc.

186. Gn. an oblique cylinder, $a b c-a_{1} b_{1} c_{1}$, with base in $H$ and a plane, $t^{\prime} T t^{\prime \prime}$, intersecting the cylinder and $\perp$ to its elements ; rq. the prs. of the intersection, mno etc., and its development in $H$ upon the trace $t^{\prime} T$.

187. Gn. an oblique circular cylinder, $a b c-a_{1} b_{1} c_{1}$, with base in $V$ and an intersecting right-line, $a b$; rq. the pts., $p, q$, in which the line pierces the surface of the cylinder.

188. Gn. an oblique circular cone, $S$-abc, with base in $H$ and an intersecting right-line, $a b$; rq. $p, q$, as in 187.

189. Gr. a sphere with centre, $c$, in neither $H$ nor $V$ and an intersecting line, $a b$; rq. $p, q$, as in 187 .

190. Gn. an oblique circular cone, $S$-abc, with base in $V$ and a pt., $p$, upon its surface; rq. the plane, $t^{\prime} T t^{\prime \prime}$, tangent to $S-a b c$ and containing $p$.

191. Gn. an oblique circular cylinder, $a b c-a_{1} b_{1} c_{1}$, with base in $H$, and a pt., $p$, upon its surface; rq. a plane, $t^{\prime} T t^{\prime \prime}$, as in 190.

192. Gn. an oblique circular cone, $S$ - $a b c$, with base in $H$ and a pt., $p$, in space; rq. the planes, $t^{\prime} T t^{\prime \prime}$, as in 190.

193. Gn. an oblique circular cylinder, $a b c-a_{1} b_{1} c_{1}$, with base in $V$ and a pt., $p$, in space ; rq. the planes, $t^{\prime} T t^{\prime \prime}$, as in 190.

194. Gn. a cone of revolution, $S$-abc, with axis $\|$ to $G$ and a pt., $p$,
a) upon its surface,
b) in space ;

rq. the tangent planes, $t^{\prime} T t^{\prime \prime}$, containing $p$.

195. Gn. a cylinder of revolution, $a b c-a_{1} b_{1} c_{1}$, with axis \| to $G$ and a direction, $m n$; rq. the tangent plane, $t^{\prime} T t^{\prime \prime}, \|$ to the given direction. 
Note. In exercises 196-9 and 202-7 the cones and cylinders are oblique, with circular bases, and mno etc., is the rq. figure of intersection.

196. Gn. a cone, $S-a b c$, with base in $H$ and a direction, $m n$; rq. the tangent plane, $t^{\prime} T t^{\prime \prime}, \|$ to the given direction.

197. Gn. a cylinder, $a b c-a_{1} b_{1} c_{1}$, with base in $V$ and a direction, $m n$; rq. the plane, $t^{\prime} T t^{\prime \prime}$, as in 196 .

198. Gn. a cylinder, $K$, with base in $H$ and the $\Varangle \epsilon$; rq. the tangent plane, $t^{\prime} T t^{\prime \prime}$, making with $H$ the $\Varangle \epsilon$.

199. Gn. a cone, $S-a b c$, with base in $H$ and the $\Varangle \epsilon ; r q$. $t^{\prime} T t^{\prime \prime}$, as in 198 .

200. Gn. a developable surface with a helical directrix and a pt., $p$, on its surface; rq. the tangent plane, $t^{\prime} T t^{\prime \prime}$, containing $p$.

201. Gn. a surface as in 198 and a line, $a b$, in space; rq. the plane, $t^{\prime} T t^{\prime \prime}$, tangent to the gn. surface and $\|$ to $a b$.

202. Gn. two intersecting cylinders, $K, L$, with bases in $H$; rq. mno etc.

203. Gn. a cylinder, $K$, with base in $V$ and an intersecting prism, $P$, with base in $H$; rq. mno etc.

204. Gn. a cylinder, $K$, and an intersecting prism, $S-a b c$, both bases in $H$; rq. mno etc.

205. Gn. a cylinder, $K$, with base in $V$ and an intersecting cone, $S-a b c$, with base in $H$; rq. mno etc.

206. Gn. two intersecting cones, $S$-abc and $X$-stu, with bases in $H ; \mathrm{rq}$. the figure of intersection, mno etc.

207. Gn. a cone, $S-a b c$, with base in $V$ and an intersecting pyramid, $X$-stuv, with base in $H$; rq. mno etc.

208. Gn. a sphere with centre, $c$, not in $H$ or $V$ and a pt., $p$, in its surface; rq. the tangent plane, $t^{\prime} T t^{\prime \prime}$, containing $p$.

209. Gn. a sphere with $c$ in $G$ and a line, $a b$; rq. the tangent planes, $t^{\prime} T t^{\prime \prime}$, containing $a b$.

210. Gn. a sphere with $c$ in neither $H$ nor $V$ and a line, $a b$; rq. the tangent planes, $t^{\prime} T t^{\prime \prime}$, containing $a b$.

211. Gn. an hyperboloid of revolution of one nappe and a pt., $p$, on its surface; rq. the tangent plane, $t^{\prime} T t^{\prime \prime}$, containing $p$. Assume the axis $\perp$ to $H$; also in the following exercises on 
surfaces of revolution. Construct the figure by revolving about the axis a line windschief with respect to it.

212. Gn. an ellipsoid of revolution and a pt., $p$, on its surface ; rq. the tangent plane, $t^{\prime} T t^{\prime \prime}$, containing $p$.

213. Gn. any surface of revolution and a pt., $p$, thereon; rq. the tangent plane, $t^{\prime} T t^{\prime \prime}$, containing $p$.

214. Gn. any surface of revolution and a line, $a b$; rq. the tangent plane, $t^{\prime} T t^{\prime \prime}$, containing $a b$.

215. Gn. an ellipsoid and a pt., $p$, without it; rq. the tangent cone having $p$ as its vertex, also rq. the curve of contact, mno etc.

216. Gn. a sphere and an intersecting oblique cylinder; rq. the figure of intersection, mno etc.

217. Gn. any two intersecting surfaces of revolution with axes intersecting; rq. the figure of intersection, mno etc.

218. Construct the general hyperboloid of one nappe, whose right section is elliptical ; also construct its asymptote cone.

219. Construct the hyperbolic paraboloid, assume a pt., $p$, upon its surface and find the tangent plane, $t^{\prime} T t^{\prime \prime}$, containing $p$.

220. Gn. three space curves, $a b, c d, e f$, as directrices of a warped surface and a pt., $p$, in $a b$; rq. the rectilinear element of the surface, $p q$, passing through $p$.

221. Gn. two space curves, $a b, c d$, as linear directrices and the plane, $t^{\prime} T t^{\prime \prime}$, as a plane directer of a warped surface; also given

a) a pt., $p$, in $a b$,

b) a line, $m n$, in $t^{\prime} T t^{\prime \prime}$, or $\|$ to it;

rq. a) $p q$, the rectilinear element of the surface passing through $p$,

b) $p q$, the rectilinear element of the surface $\|$ to $m n$.

222. Gn. a right-line, $a b, \perp$ to $H$ and the helix, $c d$, with $a b$ for its axis ; rq. to construct upon these directrices the helicoid or screw surface, the rectilinear elements making with $a b$ the $\Varangle 60^{\circ}$.

223. Gn. a helicoid and a pt., $p$, upon its surface; rq. the tangent plane, $t^{\prime} T t^{\prime \prime}$, passing through the gn. pt. 
224. Gn. an hyperboloid of revolution with axis $\perp$ to $H$ and an intersecting plane, $t^{\prime} T t^{\prime \prime} ; \mathrm{rq}$. the figure of intersection and its development.

\section{Section IV.}

Additional Exercises on Lines and Surfaces of an Order higher than the First.

225. Gn. a pt., $p$, and a line, $a b$; rq. the prs. of a circle, nno, whose centre lies in $a b$, whose circumference passes through $p$ and whose plane, $t^{\prime} T t^{\prime \prime}$, is $\perp$ to $a b$.

226. Gn. a plane, $t^{\prime} T t^{\prime \prime}$, and a pt., $S$, without it; rq. the prs. of the cone, $S$-abc, whose vertex is at $S$, whose circular base lies in $t^{\prime} T t^{\prime \prime}$ and whose elements make with its axis the \. $30^{\circ}$.

227. Gn. in $V$ a circle tangent to $H$ and in $H$ a pt., $m$; rq. a pt., $o$, so situated that the projection of the circle in $H$ from $o$ as a centre shall be an hyperbola with middle pt. $m$ and an asymptote angle of $120^{\circ}$.

228. Gn. in $V$ a circle tangent to $H$ and in $H$ a line, $a b$, or a pt., $m$; rq. a pt., $o$, from which as a centre the circle will be projected in $H$ as a parabola with $a b$ as axis or with $m$ as focus.

229. Of a conic section gn. two tangents, $a b, c d$, the pts. $m, n$, as pts. of contact for $a b, c d$, respectively and

a) another pt., $p$,

b) another tangent, $q s$;

rq. the section, its centre and principal axes.

230. Of an hyperbola there is given an asymptote, $a b$, and

a) three pts., $c, d, e$, or

b) three tangents, $m n, o p, q r$;

rq. the other asymptote, $\alpha \beta$, the principal axes and the hyperbola itself.

231. Of a conic section there is known one of the foci, $F$, and a) three pts., $a, b, c$, of the circumference, or

b) three tangents, $m n, o p, q r$;

rq. the section, its centre and its principal axes. 
232. A line, $L$, of unchangeable length glides with its end pts. in two intersecting right-lines, $a b, c d, \perp$ to each other; rq. the enveloped curve of $L$, the path of any pt., $p$, upon $L$. What is this path? Prove your answer.

233. Gn. a $\triangle, a b c$, of unchangeable form and in its plane two intersecting lines $m n$, op, with their $\Varangle=$ to the $\Varangle c$ of the $\Delta$; the $\Delta$ moves with its vertices $a, b$, in $m n, o p$, respectively; rq. the locus of $c$; of the centre, $o$, of the circumscribed circle ; of the centre of gravity, $g$, of the $\Delta$; of any pt., $x$, of the $\triangle$.

234. Gn. a quadrilateral, abcd, of changing form, the vertices $a, b$, are fixed, the sides $b c, c d, d a$, remain constant in length ; rq. the curve, mno etc., enveloped by the side $c d$, and the locus of the intersection of $b c, d a$.

235. Gn. a circle, $o$, and a pt., $p$, in its plane ; rq. the locus, mno etc., of the intersections of all $\perp$ s that can be drawn from $p$ upon the tangents of the circle; construct the tangent at any pt., $q$, and find the centre of curvature, $\rho$, for the same pt.

236. Gn. two circles, $o$ and $o^{\prime}$, in a plane and the $\Varangle \alpha$; rq. the locus of the intersection of all pairs of tangents, one from each circle, meeting at the $\Varangle a$; solve when $\alpha=75^{\circ}$ or $90^{\circ}$.

237. Gn. a right-line, $a b$, a pt., $p$, and a length, $L$; rq. the curve, mno etc., formed when $p$ is joined with every pt. of $a b$ and the length, $L$, is measured from $a b$ upon these radii vectores. Rq. the curve when $L \quad a$ ) is greater than, $b$ ) is equal to, $c$ ) is less than, the distance from $p$ to $a b$.

238. The same as 237 when a circle, $o$, is given, instead of the line $a b$.

239. Construct a cycloid, also one of its undulating trochoids and one of its trochoids with double pt., also construct the evolute of the cycloid and a tangent at any pt.

240. Construct an epicycloid, an undulating and a crossed epitrochoid $a$ ) when the radius of the fixed circle is donble that of the rolling circle, $b$ ) when the former equals the latter. Construct the evolute of the epicycloid in case $a$ ) and in 
case $b$ ) show that the epicycloid is identical with the cardioid of 235 .

241. Construct a hypocycloid, an undulating and a crossed hypotrochoid and the evolute of the first when the radius of the fixed circle is $a$ ) three times, $b$ ) four times, $c$ ) two and a half times, as great as that of the rolling circle. Show that the hypocycloid in case $b$ ) is identical with the envelope of a line of constant length moving with its ends in two right-lines $\perp$ to each other.

242. Gn. a plane, $t^{\prime} T t^{\prime \prime}$, and a pt., $p$, in $t^{\prime} T$; rq. a complete branch of a cycloid having $T t^{\prime \prime}$ for its base and tangent to $t^{\prime} T$ at the pt. $p$.

243. The axis $S o$ of a cone of the $2 \mathrm{~d}$ order is given $\|$ to $G$, the planes of the major and minor sections are $\|$ to $V$ and $H$ respectively; rq. the focus lines of the cone and the three principal projections of its intersection with a sphere whose centre is at its vertex.

244. Gn. two intersecting right-lines, $a b, c d$; through $a b$ there is laid a complete system of planes, and to each of these a $\perp$ plane containing $c d$. Examine the locus of the intersections of the pairs of $\perp$ planes, and find its intersections with $H$ and $V$.

245. Upon a sphere with centre $o$ lie two pts., $a, b$; two great circles always at right angles to each other are moved along the surface of the sphere, one always passing through $a$, the other through $b$; examine the locus on the sphere of their pt. of intersection.

246. The directrix of a cone whose vertex is $S$, is a conchoid, $a b c$, in $H$. 'This cone is cut ky a plane, $t^{\prime} T t^{\prime \prime}$, whose $t^{\prime} T$ is $\perp$ to the asymptote of $a b c$; rq. the projections of the intersection, mno, and its true form, $(m)(n)(o)$.

247. Gn. in $H$ a parabola by its focus, $f$, and principal tangent, $a b$; the locus, def, of the intersections of $\perp$ s from the principal vertex, $A$, with the tangents of the parabola, is taken as the directrix of a cone with given vertex, $S$; rq. the intersection, mno, of the cone with any plane, $t^{\prime} T t^{\prime \prime}$. 
248. Gn. an oblique cylinder with its elements \| to a gn. line, $a b$, and its directrix a common cycloid in $H$; rq. a normal section, mno, the true form of the latter, and for any pt., $p$, of mno the tangent, $p q$, and centre of curvature, $\rho$.

249. Gn. a right-line, $a b$, a cylinder, $a b c-a_{1} b_{1} c_{1}$, and a cone, $S$-def, none intersecting either of the other two;

rq. $\quad a$ ) planes $t^{\prime} T t^{\prime \prime}, r^{\prime} R r^{\prime \prime}, \|$ to $a b$ and tangent to $S$-def, and their intersections, mno, $m_{1} n_{1} o_{1}$, with the cylinder,

b) planes $t^{\prime} T t^{\prime \prime}, r^{\prime} R r^{\prime \prime}, \|$ to $a b$ and tangent to $a b c$ $a_{1} b_{1} c_{1}$, and their intersections, mno, $m_{1} n_{1} o_{1}$, with the cone.

250. Gn. a cone of revolution with the diameter of the base equal to one-half the slant height, and a pt., $a$, upon its surface; rq. the curve, $a b c a$, of shortest distance from $a$ around upon the surface of the cone back to the pt. $a$ again, and the tangent at any pt., $b$.

251. Gn. two circular cylinders $C, C_{1}$, with bases equal circles in $H$ and with elements so drawn that a plane $\|$ to both systems has its $H$ trace $\|$ to the line joining the centres of the bases; rq. the intersection, mno, of the surfaces ; project isometrically the solid thus cut ont.

252. Gn. a cylinder of revolution, $C$, and a cone of revolution, $R$, with bases in $H$, with an element of $C$ for axis of $R$, and with the plane of the axes of $C$ and $R \|$ to $V$; rq. curve of intersection, mno, and proof that its $V$-pr. is a parabola.

253. Gn. two cylinders of revolution of equal normal section which pierce each other at right angles; the axis of each cylinder is tangent to the cylindrical surface of the other; rq. the figure of intersection, mno, its isometric projection, the development of one of the cylinders, and the tangent at the pt. $p$ of the developed curve.

254. Gn. two windschief lines, $a b, c d$, and in $a b$ the pt. $p$; rq. the prs. of a helix which has $a b, c d$, for principal normals and passes through the pt. $p$. That is, $a b, c d$, cut the helix at right angles and are tangent to the cylinder of revolution upon which the helix is wound; rq. two spires of the helix. 
255. Gn. a developable helical surface;

rq. a) its intersection, mno etc., with a plane passing through the axis. Assume axis $\perp$ to $H$ and cut by a plane $\perp$ to $V$;

b) its intersection, rst etc., with any plane,

c) its development in $H$.

256. Gn. a developable or tangential helical surface and upon it two pts., $a, b$; rq. the shortest path along the surface from $a$ to $b$.

257. Gn. two cones of revolution, $C, C^{\prime}$, with elements the same length, but with the diameter of the base of $C^{\prime}$ one-half that of $C$; their vertices are made to coincide and $C^{\prime}$ is rolled externally upon $C$, the two surfaces always having one and only one element in common; rq. one complete branch, mno, of the locus of any pt., $a$, in the circumference of the base of $C^{\prime \prime}$, also corresponding conical crossed and inflected epitrochoids.

258. Gn. two windschief lines, $a b, c d$; rq. two single surfaced hyperboloids of revolution having $a b$ and $c d$ for axes and designed to work together tangentially, transmitting motions

a) equal,

$b$ ) in which the angular velocity of hyperboloid $a b$ is to that of hyperboloid $c d$ as $2: 3$.

259. Gn. in space three windschief lines, $a b, c d, e f$; rq. the hyperboloid of one nappe constructed upon these lines as directrices, its axis and the ellipse of its gorge.

260. Gn. two parabolas with the directions from their principal vertices to their foci exactly opposite and with their planes always at right angles. One parabola moves as a generatrix with its principal vertex gliding in the other parabola. All positions of the two axes are $\|$ and all positions of the plane of the generating parabola are $\|$; rq. the surface described.

261. Gn. a cone of revolution and a co-axial helicoid; rq. the intersection, mno etc., of the two surfaces.

262. Gn. two windschief lines, $a b, c d$, and a line, $L$, of limited but fixed length longer than the common $\perp$ of $a b, c d$. 
$L$ moves with one end in $a b$, the other in $c d$; rq. the surface described and its intersection, mno etc., by a plaue, $t^{\prime} T t^{\prime \prime}, \perp$ to the common $\perp$ of $a b, c d$, at its middle pt., $p$, and the development of mno etc., upon $t^{\prime} T$.

263. Gn. as meridian section of a surface of revolution two equal circles, the distance of whose centres is two-thirds the diameter of either circle, the axis of the surface being the common chord. Intersect this surface by a plane $\|$ to its axis and bisecting the radius of its equator; rq. the section, mno etc., which is a Cassinian curve.

264. Gn. a complete spire of a helix and a sphere with radius one-fourth of that of the cylinder upon which the helix is wound; rq. the surface described when the sphere moves with its centre in the helix.

265. Gn. a staircase vestibule 12 feet square and 14 feet high. A spiral staircase begius at the middle pt. of one side and ends on the floor above, after having wound through an arc of $270^{\circ}$. It leaves an opening in the form of a cylinder of revolution with axis vertical, whose shortest distance from any one of the three sides bearing the staircase is 3 feet. Each stair is 6 inches high, and a balustrade two feet high, starting from a newel post at the bottom, winds about the well-hole to the top ; rq. the projections of the staircase. Design the balustrade, its supports and the newel post according to taste. 


\title{
PART II.
}

\section{SUGGESTIONS, ANALYSES AND DEMONSTRATIONS.}

\author{
Section I. \\ Point, Line, Plane.
}

Problem 1. Figure 1 of the plates shows in isometric perspective the pt. $a$ in space in the first quadrant and its projections; Fig. 2 shows the orthographic transformation of the same. In general, a pt. will be found in some one of the four quadrants. The student will easily see the transformations to be made when the pt. lies in the second, third, or fourth quadrant. Special positions of the pt. will arise when it lies in some projecting plane, or ground-line, or plane bisecting the diedral angle between two planes of projection.

Рrob. 3. Fig. 3 shows in isometric perspective the line $a b$ crossing the first quadrant, while Fig. 4 shows the same line in projection ; it will be noted that the $H$-pr. above $G$, or to the right of $G_{2}$, is regarded as covered by $V$, respectively $P$, and is represented therefore by short dashes; similarly the $V$-pr. below $G$, or to the right of $G_{2}$, and the $P$-pr. to the left of $G_{2}$, or below $G$; for $G_{3}$ regarded as belonging to $P$ coincides after transformation with $G$. The student should be careful to designate in each drawing the pts. $h, v, p$. In the line $a b, h$ is the pt. of meeting of $H$, the $H$ projecting plane of $a b$ and its $V$ projecting plane. The last and $H$ form two intersecting planes, both $\perp$ to a third or $V$. Their line of intersection passing through $h$ is therefore $\perp$ to $V$, therefore to $G$ at the pt. where the $V$-pr. meets $G$. Therefore to find $h$ we have the directions given in Art 15, of Part III. Similarly for $v$ and $p$. Special positions of the line occur when it is $\|$ to one or more 
of the planes of projection, or lies in one or two of those planes, or in one of the bisecting planes of the diedral angles formed by any two of the fundamental planes. Rigorously taken there are eight angular spaces bounded by the three fundamental planes, but we shall understand by the four quadrants the four diedral angles formed by the intersection of $H$ and $V$.

Рвов. 4. From this point on, the third projection may be omitted except in the special cases which require its use.

Рвов. 7. Lines lying in a plane $\|$ to $P$ are not fully known unless their $P$-pr. is given or their $v$ and $h$.

Рвов. 9. The line joining the $H$ - and $V$-prs. of a pt. must always be $\perp$ to $G$.

Рвов. 11. Fig. 7 represents a cone of revolution with base in $H$ and axis in $V ; a^{\prime}$ is the inclination of the elements to $H$. Fig. 8 represents a cylinder of revolution with base in $H$ and the axis not in $V$. Fig. 9 represents a sphere with centre in $G$. Fig. 10, a sphere with centre in space in the first quadrant. Simple changes of these figures in position will solve problems 11 and 12 .

Note. The student will find that the ease with which a problem is solved depends greatly upon the skill with which the data is assumed. Study the simplest representations consistent with generality. Work also in the various quadrants and with various positions.

Proв. 13. Take $a^{\prime} b^{\prime}$ between $h$ and $G$ as the base of a right $\Varangle \mathrm{d} \Delta$, and the distance from $v$ to $G$ as the $\perp$. That is, from the pt. where $a^{\prime} b^{\prime}$ cuts $G$ draw in $H$ an indefinite $\perp$ to $a^{\prime} b^{\prime}$. With the same pt. in $G$ as a centre and its distance to $v$ as a radius, describe a circle till it intersects the $\perp$ drawn to $a^{\prime} b^{\prime}$ in $H$. This pt. of intersection joined to $h$ gives $L$ and its inclination to $a^{\prime} b^{\prime}$ gives $a^{\prime}$. Proceed in the same way with $a^{\prime \prime} b^{\prime \prime}$.

Prob. 14. Since the line connecting two points in space is the hypotenuse of a right $\Varangle \mathrm{d} \Delta$, of which the distance between the $H$ or $V$-prs. is the base and the difference between the $V$ or $H$ projecting lines the $\perp$, it is sufficient to construct a right $\Varangle \mathrm{d} \Delta$ with these legs. Or one pr. of the connecting line $a b$ can be revolved about one of its extremities till $\|$ with $G$, when 
the line in its new position will be $\|$ to the other plane and its new projection in that plane will be its true length.

Prob. 17. One solution is shown in Fig. 11.

Рroв. 19. The $\Varangle$ between the $H$-pr. and $G$ is the pr. of $a^{\prime \prime}$. Construct an $\Varangle$ equal to $a^{\prime \prime}$ and $\|$ to $H$, one side being in $V$ and vertex at $v$. Revolve this $\Varangle$ about a $\perp$ to $V$ through $v$ as an axis, until its $H$-pr. as shown by some pt. in the line coinciding with a pt. of the side of the $Y$, coincides with the given $H$-pr. The $V$-pr. belonging to this position fully determines the line.

Рвов. 20. Construct a rectangular parallelopiped with one face in $H$ and one in $V$, such that the diagonal makes the $\Varangle a^{\prime}$ with the diagonal of the face in $H$ and the $\Varangle a^{\prime \prime}$ with the diagonal of the face in $V$. Pass a $\|$ line through the pt. $h$.

Рвов. 21. Solve by first finding $p^{\prime \prime \prime}$, using as an auxiliary line the bisector of the $\Varangle$ between $G_{2}$ and $G_{3}$.

Рвов. 22. The representation of a plane in space and in projection is shown in Figs. 5 and 6 . It will be observed that when a line lies in a plane its traces, $h, v, p$, must always be found in the corresponding traces of the plane.

Рroв. 23. Use as an auxiliary figure any line of the plane containing the required $\mathrm{pt}$.

Рвов. 24. Use as auxiliary figures planes $\perp$ to $t^{\prime} T$ and $T t^{\prime \prime}$. Revolve the intersections upon the $H$ and $V$ traces of these planes into $H$ and $V$ respectively. Fig. 6 shows how $\epsilon^{\prime}$ may be found. A similar construction should be used for $\epsilon^{\prime \prime}$.

Рвов. 27. Construct a right cone of revolution with axis in $V \perp$ to $H$ and elements making an $\Varangle \epsilon^{\prime}$ with $H$; a plane tangent to this cone makes the required $\Varangle$ with $H$. Upon the elements lying in $V$ construct a right $\Delta$ right-angled at $G$ and with opposite acute $\zeta=$ to $K$; the hypotenuse will be the length of the $V$ trace between the vertex of the cone and $G$, while the $I$ trace will be tangent to the circular base of the cone.

Рrob. 28. Find the vertex of a cone of revolution whose axis is in $V$, base in $H$, inclination of elements to $H, \epsilon^{\prime}$, and to 
whose base $t T^{\prime \prime}$ is tangent. $T t^{\prime \prime}$ connects the vertex of this cone with the pt. in which $t^{\prime} T$ intersects $G$.

Prob. 29. The auxiliary cone now has its axis in $H$ and base in $V$.

Рвов. 30. If a sphere be taken with centre in $G$ and two cones of revolution tangent to this sphere, one with vertex in $V$, axis $\perp$ to $H$ and elements making $\Varangle \epsilon^{\prime}$ with $H$, the other with vertex in $H$, axis $\perp$ to $V$ and elements making $\Varangle \epsilon^{\prime \prime}$ with $V$, a plane tangent to both cones, i.e. whose traces pass through the vertices of the cones and are tangent to their bases will be II to the plane sought. The construction is given in Fig. 12 .

Рвов. 31. The revolution upon $t^{\prime} T$ rq. is shown in Fig. 6 . Let the student solve the problem when the pt. alone is developed. He should also solve the problem when $K$ is greater than $90^{\circ}$.

Рrob. 33. Revolve into $H$ upou the line $(p) p^{\prime}$ as an axis the rt. $\Delta p^{\prime}(p) p^{\prime \prime}$. It will be seen that the basal $\Varangle$ of this rt. $\Delta=\frac{1}{2} \epsilon^{\prime}$. Therefore bisect its hypotenuse by a $\perp$; the rq. $t^{\prime} T$ must pass through the intersection of the $\perp$ with the base of the $\triangle$ and must be perpendicular to that base.

Prob. 35. The direction of the $H$ trace is known, and since with the gn. $\Varangle K$ we can construct an auxiliary plane $\|$ to the rq. one with its $H$ trace passing through the developed pt., $\epsilon^{\prime}$ is known and the problem reduces to 29 .

Рвов. 36. When the $\Varangle K$ is developed into $H$ or $V$, the loci of the developed position of the rq. pt. will be lines $\|$ to the $H$ and $V$ traces at the distances $m^{\prime}, m^{\prime \prime}$, respectively. The intersection of these loci will be the developed pt.

Рвов. 38. The line joining the $H$ traces or $V$ traces of the lines will be the $H$ or $V$ trace respectively of the plane. The $\Varangle \delta$ is found by developing the plane and lines in $H$ or $V$.

The data necessary for determining a plane are given in Art. 19 of Part. III.

Рrob. 39. Develop the given plane and line, make the rq. construction and find the rq. line by counter-revolution. 
Рrов. 40. The first part is solved the same as the first part of 38 ; the latter part by development.

The student should prove that the projections of two parallel lines are respectively parallel lines.

Риов. 41. Fig. 13 gives the solution.

Рroв. 42. 'The rq. plane is found by connecting the gn. pt. with any pt. of the gn. line and thus reducing that part of the problem to 38 .

Pros. 44. The gn. pt. is one pt. in the rq. line. With the distance of the $V$-pr. of the pt. from $G$ as a $\perp$ and $\delta$ as a basal angle form a rt. $\triangle$. With the base of this as a radius and the $H$-pr. of the pt. as a centre, describe the base of a cone of revolution. Its intersection with the $H$ trace of the gn. plane is a second pt. in the rq. line.

Prob. 45. $t^{\prime} T$ must pass through $h$ and must be tangent to the base of a cone of revolution whose axis is the $\perp$ from $v$ to $G$ and one of whose elements is the $\perp$ from $v$ upon $t^{\prime} T$.

This element is found by revolving $a b$ upon $a^{\prime} b^{\prime}$ into $H$, constructing through $h$ a line making the $\Varangle \delta$ with $a_{1} b_{1}$ and taking the perpendicular from the revolved position of $v$ to this line. Knowing the axis and element of the auxiliary cone, the radius of the base is easily found. With this radius and with the centre at the foot of the $\perp$ from $v$ to $G$, a circle is constructed. The rq. $H$ trace passes through $h$ and is tangent to this circle. The $V$ trace passes through $v$.

Рвов. 48. Construct the line cut out from the gn. plane by the $H$ or $V$ projecting planes of the line. The intersection of the $V$-pr. or $H$-pr. of this line with the $V$-pr. or $H$-pr. of the given line must indicate an actual intersection in space and be one pr. of the pt. sought. When the gn. line lies in a plane $\perp$ to $G$, its $h$ and $v$ must be given and the problem is solved by using this $\perp$ plane as a third plane of projection. Fig. 14 gives the first of the four rq. cases when $K$ is obtuse. $m^{\prime \prime} n^{\prime \prime}$ is the $V$-pr. of the line of $t^{\prime} T t^{\prime \prime}$ cut out by the $H$ projecting plane of $a b . \quad q^{\prime} r^{\prime}$ is the $H$-pr. of the line of $t^{\prime} T t^{\prime \prime}$ cut out by the $V$ projecting plane of $a b$. 
РRов. 50. Since the gn. line is $\perp$ to the rq. plane, the directions of the rq. traces are known by Part III., Art. 24. Through the gn. pt. $p$ draw a line $\|$ to the $H$ or $V$ trace of the rq. plane. This auxiliary line must be a line of the rq. plane and the pt. where it pierces the $V$ or $H$ plane must be a pt. in the $V$ or $H$ trace of the rq. plane. One pt. in either trace being known, the plane is known.

Рrob. 51. The $h$ or $v$ traces of the gn. line and the constructed $\perp$ determine the $H$ or $V$ trace of a plane; this trace may be used as an axis upon which to revolve the angle of the lines $a b$ and $p h$ into $H$ or $V$.

Рвов. 53. The rq. line nust be an element of the cone of revolution whose vertex is at the gn. pt., whose axis is $\perp$ to $H$ and whose elements make the $\Varangle \delta$ with $H$. Construct such a cone. Then to determine which element, pass a line through the vertex of the cone $\perp$ to the gn. plane. Find the pt. in which it pierces $H$. 'This $\perp$ must make with the rq. element an $\Varangle=90^{\circ}-\theta$. Construct a $\Delta$, one of whose sides shall be the true length of the $\perp$ to $t^{\prime} T t^{\prime \prime}$ from $p$ to $H$; the second, the true length of an element of the cone; the included $\zeta=90^{\circ}-\theta$. The third side is the distance from the foot of the $\perp$ to the foot of a rq. element. How many solutions?

Prob. 54. When the $H$ and $V$ traces respectively intersect within the limits of the paper, their pts. of intersection are the $h$ and $v$ of the rq. line of intersection. When the $H$ and $V$ traces do not so intersect, pass auxiliary planes $\|$ to $V$ and at such a distance that the $V$-prs. of the lines cut from the gn. planes do intersect within the necessary limits. Two of these intersections will determine the $V$-pr. of the line of intersection. A similar construction will determine its $H$-pr. Other auxiliary constructions will occur to the thoughtful student.

Prob. 55. Draw the $H$ trace of an auxiliary plane $\perp$ to the line of intersection of the two planes. In order to determine by revolution upon this $H$ trace the plane angle of the two planes, it only remains to find where the line of intersection pierces the auxiliary plane. 
Revolve the line of intersection upon its $H$-pr. into $H . \quad \mathrm{A} \perp$ let fall upon the revolved line of intersection from the pt. of intersection of the auxiliary $H$ trace with the $H$-pr. of the line of intersection, will determine the required pt. Revolve the auxiliary plane upon its $I I$ trace into $I I$. The developed pt. of piercing joined with the pts. in which the auxiliary $H$ trace crosses the $H$ traces of the given planes, will form the angle sought. A similar construction may be made in $V$.

Prob. 56. Assume a third plane $\perp$ to $H$ or $V$ and the $H$ or $V$ traces of the gn. planes. Revolve this auxiliary plane upon its $H$ or $V$ trace into $H$ or $V$. The distance of the lls cut out is the required distance.

Prob. 57. Solved by an auxiliary line through a pt. in $a b$ and $\|$ to $c d$, thus giving two intersecting lines to determine the rq. plane.

Рroв. 58. Two auxiliary lines are passed through the gn. pt., $p, \|$ to the gn. lines, $a b$ and $c d$.

Рrob. 59. If through $a b$ a plane be passed $\|$ to $c d$, by 53, and $c d$ be projected upon this plane, it is evident that the pt. in which this pr. cuts $a b$ is the foot of the common $\perp$ of the two lines.

Therefore, as in $5 \bar{\imath}$, construct a plane containing $a b$ and $\|$ to $c d$. From any pt. of $c d$ diaw a $\perp$ to the plane and find its foot. The line through this foot $\|$ to $c d$ is the pr. of the latter upon the plane and the pt. where this pr. intersects $a b$ is the foot of the rq. common $\perp$. The latter is found by drawing from its foot $\mathrm{a} \perp$ to the plane and limiting this $\perp$ by the plane and $c d$.

Prob. 60. a) If through the gn. pt. and each of the gn. lines planes be passed, their intersection will be the rq. line.

$b$ ) 'Through $p$ and any pt. of $a b$ pass an auxiliary line $m n$. Find the pts. where $a b$ and $m n$ pierce the $H$ projecting plane of $c d$, call these pts. $x, y$, respectively. The line $x y$ must be the intersection of the plane of $a b$ and $m n$ with the $H$ projecting plane of $c d$. The intersection of $x^{\prime \prime} y^{\prime \prime}$ with $c^{\prime \prime} d^{\prime \prime}$ must be the $V$-pr. of the pt. $q$, where $c d$ pierces the plane of $a b, m n$. Join $p$ and $q$. Find a proof of your work. 
Prob. 63. Draw through $p$ a line $\perp$ to $t^{\prime} T t^{\prime \prime}$ and another line $\|$ to the given line. These two intersecting lines will determine $r^{\prime} R r^{\prime \prime}$.

Рвов. 64. Consider the gn. line the line of intersection between the gn. and rq. planes and reverse the process of 55 .

Рвов. 65. If any pt. of the gn. line be chosen except its foot in the gn. plane and this pt. be taken as the vertex of a cone of revolution whose axis is $\perp$ to the gn. plane and whose elements make with that plane the $\Varangle \phi$, it is evident that the tangent to the base of this auxiliary cone from the pt. where the gn. line pierces the gn. plane, will be a second line for the determination of the rq. plane. Therefore, find the pt. where the gn. line pierces the gn. plane. Choose another pt. of the line and find where the $\perp$ from this pt. to the gn. plane pierces the latter and the length of that $\perp$. The latter with the $\Varangle \phi$ gives the radius of the base of the auxiliary cone of revolution. Develop the plane with the pts. found into $H$ or $V$. Construct the base of the cone and the rq. tangent. By counter-revolution the two necessary intersecting lines will appear.

Рвов. 66. Consider the gn. pt. the vertex of a cone of revolution whose axis is $\perp$ to the gn. plane and whose elements make the $\nvdash \delta$ with that plane. Find the axis of this cone, the radius of its base and the pt. where its axis pierces the gn. plane. In the developed position of the gn. plane find the pt. in which the base of the cone cuts the gn. line. By counterrevolution two pts. will appear for determining the rq. line. In general, two solutions.

Рвов. 67. Designate the face $\Varangle \mathrm{s}$ by $\alpha, \beta, \gamma$, their opposite dihedral $\Varangle \mathrm{s}$ by $A, B, C$. Assume one face $\Varangle$, as $\beta$, in $H$ with the edge between $a$ and $\beta \perp$ to $G$. Construct the $\Varangle \alpha$ in $H$ adjacent to and on one side of $\beta$; the $\Varangle \gamma$ in $H$ adjacent to and on the other side of $\beta$. Let the common vertex of $\alpha, \beta$, $\gamma$, be $O$. This will be the vertex of two cones of revolution, any pt. besides $(0)$ in whose common element is sought. To obtain a second pt. we determine where this common element - the edge between $a$ and $\gamma$-pierces $V$. The locus of this 
pt. in $V$ on the side of $a$ is a circle, the base of a cone of revolution, whose axis is on one side of the $\Varangle a$ and generating element the other side. If the length of this generating element be laid off on the outer side of the $\Varangle \gamma$, the distance from its extremity to the intersection in $G$ of the edge between $\beta$ and $\gamma$ will be the distance from the latter pt. to the rq. pt. in $V$. The $\Varangle C$ will be directly gn., while the $\Varangle \mathrm{s} A$ and $B$ are found by methods already explained.

This problem is constructed in Fig. 15.

Рвов. 68. Let $a, \beta, C$, be given in positions described in 67 ; the trihedral is easily found by a slight modification of the same problem.

Рвов. 69. Let $\alpha, \beta, A$, be the three parts given as above. By reversing the operation of finding $A$ in 68 the $V$ trace of the face $\Varangle \gamma$ will be known, and if the data are so taken that the problem is possible, the pts. (in general two) of the intersection of the third edge with $V$ are known.

Рвов. 72. Place the $\Varangle A$ in position, its edge being in $H \perp$ to $G$. There will then be two planes in position, one in $H$ containing the yet unknown face $\Varangle \beta$, another $\perp$ to $V$, making the $\Varangle A$ with $H$ and containing the unknown face $\Varangle \gamma$. Tangent to the latter plane construct an auxiliary sphere with its centre in $G$. Tangent to this sphere construct two cones of revolution with axes in $V$, the elements of one making with $H$ the $\Varangle C$, the elements of the other making with the plane of $\gamma$ the $\Varangle B$. Both vertices of these cones must lie in $V$ and must be pts. in the trace of the rq. plane. The $V$ trace of the latter is therefore known, and also its $H$ trace, since this is tangent to the base of the first auxiliary cone. Find $a, \beta, \gamma$.

Prob. 73. Construct an auxiliary trihedral with $\alpha$ and $(\beta+\gamma)$ adjacent face $\Varangle \mathrm{s}$ and $C$ the included dihedral. Let $(\beta+\gamma)$ be taken in $H$ and the edge of the dihedral be $\perp$ to $G$. Lay off from $O$ upon the outer side of $(\beta+\gamma)$ a line equal to the outer side of $a$ between $O$ and $G$. A line joining the pt. thus determined with the $v$ of the upper edge of the auxiliary trihedral will form the base of an isosceles triangle. A plane through 
$O \perp$ to this base gives the necessary division of $(\beta+\gamma)$. Why?

Prob. 74. This problem is an easy modification of 73 .

Рвов. 75. In finding the $V$-pr. of the pentagon it will be most direct to use as auxiliary lines of the plane those whose $H$-prs. pass through $a^{\prime}, b^{\prime}, c^{\prime}, d^{\prime}, e^{\prime}$.

To find the true figure, take a line $\|$ to $H$ through some convenient vertex of the $V$-pr. Join the corresponding pts. in $H$. This will be an axis upon which the figure is to be revolved until it is $\|$ to $H$, when it will be projected in $H$ in its true magnitude.

Рвов. 76. Find the $V$-pr. of the pts. $d$, $e$, by using the Axis of Affinity of the two prs. of the pentagon. This axis may be defined as follows: when two plane figures are so related that the intersections of homologous sides lie upon one right line, the latter is called an Axis of Affinity. The most general proof for its existence depends upon the following theorem of Desargues :-

"If each of two triangles has one vertex in each of three concurrent lines, then the intersections of corresponding lines lie in a line, those sides being called corresponding which are opposite to vertices on the same line."

This proposition is conveniently demonstrated by the methods of Projective Geometry. Such a demonstration ${ }^{1}$ may be found on page 394 of the tenth volume of "Encyclopedia Britannica."

In Fig. 16 let $A B C, A^{\prime} B^{\prime} C^{\prime}$, be two $\triangle$ with lines $a, b, c$, through homologous vertices meeting in $S$; then the homologous sides meet in some right line as $S_{1}, S_{2}, S_{3}$. This will still be true when $S$ retreats to infinity and $a, b, c$, become parallel lines, as always occurs in any two orthographic prs. of a plane figure. It also follows that for any pr. of a plane figure and the corresponding development of the figure, the axis of development must be an axis of affinity.

Prob. 76 is solved in Fig. 17.

${ }^{1}$ See also Chauvenet's Geom. p. 342. 
РRов. 77. Let the side of known length be the side $a b$, having an extremity in the vertex $\alpha$. The $H$-pr. and true length of this side being known, its $V$-pr. is known and the $Y$ it makes with $H$. Pass an auxiliary plane through $a \perp$ to $a b$. The inclination of this plane to $H$ is the complement of the inclination to $H$ of $a b$. The other side, $a d$, having an extremity in the vertex $a$, must lie in this plane. By developing the latter on its $H$ trace the true length of $a d$ is known and thus the whole figure.

Рвов. 78. Construct the surface right line passing through the vertex and the $V$-pr. of the pt. The $H$-pr. must lie in this line. The base of the pyramid is assumed in $H$. The construction is carried out in Fig. 18.

Рвов. 79. With the vertex as a centre revolve the $V$-pr. of the lines forming the edges until they are $\|$ to $H$, when the $H$-prs. will be the true length and will show the inclinations. In the development place the triangles forming the lateral surface adjacent to each other in $V$. The inclinations of the faces to each other are found by 55 .

Prob. 80. Construct the lateral faces in $H$, each upon its basal side. The vertex of the tetrahedron is found by revolving any two of the developed lateral faces upon their bases until their vertices unite.

Рroв. 81. Take the base in $H$ and construct adjacent to this in its proper position the developed face $s a b$, whose edges are known. The $H$-pr. of the vertex $s$ must lie in the $\perp$, or the $\perp$ produced, drawn from the vertex of this face to its base, $a b$. The $\perp$ itself is the hypotenuse and the gn. altitude the $\perp$ of a rt. $\triangle$ whose base is the distance from $a b$ to the $H$-pr. of $s$.

Рroв. 82. Place the three face $\Varangle_{\mathrm{s}} \alpha, \beta, \gamma$, adjacent in $H$. The bases of the triangular faces in the developed position must all be tangent to the circle whose centre is at the developed vertex and whose radius is the $\perp$ distance from this centre to any basal edge. Therefore each face $\Varangle$ must be divided into two segments, each of which must be equal to the divisions 
lying adjacent in the adjacent face $\Varangle$. Let the divisions of $a$ be $x$ and $y$. These two equations must then be true:

$$
x+y=\alpha, \beta-x=\gamma-y . \quad \therefore x=\frac{a+\beta-\gamma}{2}, y=\frac{a-\beta+\gamma}{2} .
$$

The known base is then introduced $\perp$ to the dividing line of its face. The construction for the remaining two bases easily follows. In dividing a care must be taken to place the greater of the two divisions adjacent to the greater of the two remaining face $\Varangle$ s.

Рвов. 83. The dihedrals can be found by Prob. 67. If the known lateral edges and inclination belong to the same face, the base is directly introduced. If not, the problem is reduced to 65 .

Рrob. 84. Take the base in $H$. The centre of the circumscribed sphere must lie in the $\perp$ erected to the base at its middle pt. It must also lie in a plane constructed $\perp$ to a lateral edge at its middle pt. It must, therefore, be the pt. where the $\perp$ pierces the plane. The radius is the distance from the centre thus found to any one of the vertices of the tetrahedron.

Prob. 85. Take in $H$ one face which we will call the base. Conceive three planes to be passed bisecting the basal dihedral angles of the tetrahedron. A new tetrahedion is thus formed whose vertex is the rq. centre. To find the auxiliary solid, intersect both tetrahedrons by a plane $\|$ to $H$. By means of planes $\perp$ to the basal edges construct plane $\chi_{\mathrm{s}}$ measuring the basal dihedrals of the gn. tetrahedron and revolve these into $H$ together with the lines cut out by the plane \| to $H$. If through the pts. where the bisectors of these $\Varangle \mathrm{s}$ pierce the || plane lines be drawn I| respectively to the basal edges of the tetrahedron, two pts. will be known in each of the three lateral edges of the auxiliary tetrahedron, therefore its vertex (the rq. centre) will be known and the rq. radius.

Рвов. 86. In each construction assume a face in $H$, one of whose sides is the gn. edge, $a b$, while no side of the base is $\|$ or $\perp$ to $G$.

a) The construction of the regular tetrahedron presents no difficulty. In this and succeeding solids consider each pr. by 
itself and use short dashes for those prs. where the lines in space are hidden or covered by the solid.

$b$ ) It will readily be seen that the boundary of the $H$-pr. of the regular octahedron is a regular hexagon, the $V$-pr. a parallelogram.

c) Note first that there are four sets of vertices, three in each set, at four different altitudes, found therefore in $V$ in four different rows. Then note that each set are the vertices of a regular $\Delta$, that a $\perp$ erected to the base in $H$ at its middle pt. passes through the centre of all the $\triangle$ named and, therefore, that all the vertices fall in $H$ in two concentric circles, forming therein the vertices of two similarly situated regular hexagons. To find the radius of the larger circle note that the three vertices nearest the base are the vertices of three regular pentagonal pyramids, the bases of which may be represented in $H$, each adjacent to a different side of the base of the solid. From this position revolve the pentagons upon the adjacent sides of the base of the solid as axes until the projected vertices of the pentagon nearest the triangular base meet in pairs upon the produced medial lines of the latter. Any one of the pts. thus described determines the radius sought. The lowest three vertices lie in $H$; therefore their $V$-prs. are in $G$. 'The next set are the vertices of the pentagonal pyramids mentioned above. If the altitude of the fundamental $\Delta$ be taken as an hypotenuse and the altitude of the $H$-pr. of a $\triangle$ adjacent to the base be taken as the base of a rt. $\Delta$, the $\perp$ of the latter will be the distance of the second set of vertices above $G$. The second vertical distance is found in a similar way, while the fourth set of vertices are as high above the second as the third are above the first. Fig. 19 gives a convenient method for describing a regular pentagon when the length of one side $A B$ is given. Give the algebraic proof that it is correct. The construction for the icosahedron is given in Fig. 20 .

d) The method of procedure with the dodecahedron is very similar to that of the icosahedron, except the work is somewhat simpler. The chief difference is found in the fact that the 
$H$-pr. of the vertices of the former are the vertices of two regular decagons.

Рвов. 87. The direction of $a c$ is found by Prob. 20. The direction of the adjacent edge, $a b \|$ to $H$, can be found by Prob. 45, the gn. $\Varangle$ being an $\Varangle$ of a regular pentagon. These two lines determine the position of a plane upon which an icosahedron satisfying the conditions may be placed. Develop the plane in $H$. Construct a regular icosahedron with upper face in $H$, one side of this face being assumed $\|$ to the $H$ trace of the plane. The counter-revolution solves the problem. This operation is readily effected by utilizing the fact that the vertices lie in four planes whose distances apart are known.

Рroв. 89. Find the pts. where the edges of the pyramid pierce the plane and join these in their proper order being careful to observe the rule with reference to covered lines, or find the lines in which the planes of the faces intersect $t^{\prime} T t^{\prime \prime}$. Portions of the lines form the sides of mno.

Рroв. 92. Join the vertex $S$ with any pt. of de. These intersecting lines determine an auxiliary plane. If the line pierces the pyramid and the base of the latter is in $\mathrm{H}$, then the $H$ trace of the auxiliary plane cuts the base, $a b c$, in two points which are to be joined to $S$. The lines thus determined are the section of the auxiliary plane and pyramid and cut the gn. line in the rq. pts., $m, n$.

Рroв. 93. Proceed in this problem as in 92, except that the auxiliary plane is determined by passing through any pt. of de a line $\|$ to the lateral edges of the prism.

Prob. 94. The auxiliary planes of simplest section must pass through both vertices. Therefore determine the pt. in which a line joining the vertices $S$ and $T$ pierces $H$. The $H$ traces of auxiliary planes are then passed through this pt. and the foot pts. of the edges of the intersecting surfaces. If any one of these planes cuts both solids there will lie in it right lines passing through $S$ and $T$, cut from both surfaces, and their mutual intersections will be the salient pts. in the rq. figure, mno etc. Only those portions of the intersection are 
to be represented as visible in either pr. which are visible in each solid taken independently.

Prob. 95. Through any pt. in space pass two lines $\|$ respectively to the edges of $a b c d-a_{1} b_{1} c_{1} d_{1}$ and $x y z-x_{1} y_{1} z_{1}$. The $V$ traces of auxiliary planes to be used as in 94 will be $\|$ to the $V$ trace of the plane of these two lines.

Рвов. 96. A construction similar to the one required is given in Fig. 21. A line is passed through the vertex $S$ parallel to the lateral edges of the prisin. The $H$ and $V$ traces of the rq. auxiliary planes pass through $h$ and $v$, respectively, of this line.

Рrob. 97. Put the plane in position and construct unit rectangular axes $\|$ to $G_{1}, G_{3}, G_{2}$, respectively. From the extremities of these axes let fall $\downarrow$ s upon the plane and find the pts. of piercing. Develop the plane together with these pts. into $H$.

Рвов. 98. Every face of a regular dodecahedron is pierced at its vertices by five edges of the solid, one at each vertex. If these edges be produced, they meet in vertices of regular pentagonal pyramids over the centres of the several faces. The entire figure thus derived is the star-dodecahedron and the new vertices are the vertices of a regular icosahedron. In a similar way the regular dodecahedron may be formed about the icosaluedron. These two solids are therefore cailed reciprocal. To project the star-dodecahedron as rq., the directions of the axes are first found by assuming $o z$ in any convenient position. Lay off, then, from $o$ two axes whose directions make with $o z \quad \chi_{\mathrm{s}}=$ to $90^{\circ}-\arctan \left(\frac{1}{11}\right)$ for $o x$ and $90^{\circ}-\arctan \left(\frac{1}{3}\right)$ for $o y$. Construct the dodecahedron orthographically with one edge $\|$ to $G$. The vertices of the rq. figure are found by taking the $z$ distances without change; while for the new $x$ and $y$ distances $\frac{9}{10}$ and $\frac{1}{2}$, respectively, of the old distances are taken.

Fig. 22 shows the axonometrical pr. of a cube in the system given above. The meaning of this pr. is this: a plane is inclined to the regular planes of pr., as in 84 , and is so taken 
that the orthographic pr. upon it of any figure in its simple descriptive position closely resembles central perspective and for mechanical purposes can be used in place of the latter. The most common form is the so-called isometric pr. In this the unit rectangular axes remain equal lines and make angles with each other of $120^{\circ}$. Figs. 1, 3, 5 are examples of its use.

The six exercises which follow are closely connected with the branch of Descriptive Geometry here referred to and may be employed in determining the positions and relative projected lengths of unit rectangular axes under given conditions.

Рвов. 99. On any side of the $\triangle a b c$, in Fig. 23, as $b c$, lay off the $\Delta a^{\prime} b c$ similar to the $\Delta m n o$ and draw the circle $K$ passing through $a a^{\prime}$ and having its centre in $b c . K$ cuts $b c$ in two pts., $d^{\prime}, e^{\prime}$, which form with $a$ and $a^{\prime}$ two rt. $\Delta$, dae and $d a^{\prime} e$, whose acute angles are of different magnitudes. Let the $\Varangle$ aed be greater than the $\Varangle a^{\prime} e d^{\prime}$. We take ae as the axis of affinity $E$, assume the projecting rays $\perp$ to $E$, lay off the $\Varangle a e d^{\prime}=a$ in $a^{\prime} e d$ and take the ratio of foreshortening $=\frac{a d^{\prime}}{a d}$.

In the pr. system so determined the $\triangle a b^{\prime} c^{\prime}$, corresponding to the $\Delta a b c$, is the $\Delta$ rq. For the rt. $\Delta a^{\prime} e d$ and $a e d^{\prime}$ are similar; therefore the $\Varangle a^{\prime} d e=\Varangle a d^{\prime} e$ and we have the continued proportion: $a^{\prime} d: d e: d c: d b=a d^{\prime}: d^{\prime} e: d^{\prime} c^{\prime}: d^{\prime} b^{\prime}$. Therefore the $\triangle a^{\prime} d e$ and $a d^{\prime} e$ are similar; hence $a^{\prime} b c$ and $a b^{\prime} c^{\prime}$ are similar.

Рroв. 100. The solution is shown in Fig. 24. Let $a^{\prime} b^{\prime} c^{\prime}$ be the given $H$-pr. of the $\triangle a b c$. Upon its base $b^{\prime} c^{\prime}$ construct $d b^{\prime} c^{\prime}$ si nilar to mno. With centre in $b^{\prime} c^{\prime}$ construct the circle $K$ passing through $a^{\prime}$ and $d$ and intersecting $b^{\prime} c^{\prime}$ in $e$ and $f$. Complete the right $\triangle a^{\prime} f e$ and $d f e$. Conceive $f$ to move in a line $\perp$ to $H, a^{\prime} e$ being taken as the axis of affinity, $E ; b^{\prime}$ and $c^{\prime}$, as vertices of the $\triangle a^{\prime} b^{\prime} c^{\prime}$, will also move in a line $\perp$ to $H$, while the same pts., as vertices of the $\Delta d b^{\prime} c^{\prime}$, will remain fixed. When $a^{\prime} f e$ is similar to $d f e$, then will $a b c$ in space be similar to $d b^{\prime} c^{\prime}$. Therefore ccnstruct the $\Delta a_{1} e_{1} f_{1}$ upon $a_{1} e_{1}=a^{\prime} e$ and similar to def; then will $a_{1} f_{1}$ be the hypotenuse and $a_{1} f_{11}=\alpha^{\prime} f$ the base 
of a rt. $\triangle$ whose perpendicular $f_{11} f_{111}$ will be the altitude above $H$ of the vertex $f$ of the $\triangle a f c$ when $a$ remains in $H$. Thence can be easily deduced the corresponding altitudes of $b$ and $c$.

Рвов. 101. The proof for the following method is by quaternions and may be found in the seventeenth volume of "Zeitschrift für mathematischen und naturwissenschaftlichen Unterricht," page 481. In Fig. 25, let $O^{\prime \prime} A^{\prime \prime}, O^{\prime \prime} B^{\prime \prime}$, be the $V$-pr. of two concurrent edges of a cube. Make $O^{\prime \prime} A^{\prime \prime} M$ similar to $O^{\prime \prime} A^{\prime \prime} B^{\prime \prime}$ and complete the $\square O^{\prime \prime} M N B^{\prime \prime}$. It will be noticed that $O^{\prime \prime} A^{\prime \prime}=\sqrt{O^{\prime \prime} B^{\prime \prime} \times O^{\prime \prime} M}$. Lay off $O^{\prime \prime} P=$ to $O^{\prime \prime} N$, but in the opposite direction. Bisect $P O^{\prime \prime} B^{\prime \prime}$ and lay off $O^{\prime \prime} C^{\prime \prime}$ $=\sqrt{O^{\prime \prime} P \times O^{\prime \prime} B^{\prime \prime}} . \quad O^{\prime \prime} C^{\prime \prime}$ will be the quantity sought. It will be observed that these axes, when taken positively and negatively through $O^{\prime \prime}$, form the concurrent edges of eight equal cubes.

Probs. 102, 103 and 104. These are instructive variations of 101 .

\section{Section II.}

Lines and Surfaces of an Order higher than the First.

Рвов. 171. a) For the ellipse take in Fig. 26 the medial lines $A A^{\prime}, B B^{\prime}$, of the $\square$ as axes. Draw lines $C N \|$ to the diagonal $D O$. Connect pts. $C$ with $A$, pts. $N$ with $A^{\prime}$. The intersections $P$ of corresponding lines are pts. upon the rq. inscribed ellipse and $B B^{\prime}, A A^{\prime}$, are conjugate diameters. For, draw $P M, P F$, \| respectively to $B B^{\prime}, A A^{\prime}$.

Let $O A=a, O B=b, O M=x, O F=y$;

$$
\begin{aligned}
& \frac{O N}{O B^{\prime}}=\frac{C D}{O A} . \quad \therefore O N=q b, C D=q a . \\
& \frac{O N}{A^{\prime} O}=\frac{M P}{A^{\prime} M} . \quad \therefore \frac{q b}{a}=\frac{y}{a+x} . \\
& \frac{O B^{\prime}}{C D}=\frac{M P}{M A} \cdot \quad \therefore \frac{b}{q a}=\frac{y}{a-x} .
\end{aligned}
$$


Multiplying the first result by the second, member for member,

$$
\frac{b^{2}}{a^{2}}=\frac{y^{2}}{a^{2}-x^{2}} . \quad \therefore a^{2} y^{2}+b^{2} x^{2}=a^{2} b^{2},
$$

the well-known equation of the ellipse.

b) For the hyperbola take axes in Fig. 27 as in the ellipse, draw lines $N N^{\prime} \|$ to the diagonal $O E$; through the pts. $N^{\prime}$ draw lines $N^{\prime} C \|$ to the other diagonal $O D$. The intersections $P$ of lines $A^{\prime} N$ with their corresponding lines $A C$ are pts. of an hyperbola ; for, let $O A=a, O B=b, O M=x, O F=y$;

$$
\begin{aligned}
& \frac{O N^{\prime}}{O A}=\frac{D C}{O A}=\frac{O N}{O B} . \quad \therefore C D=q a, \quad O N=q b . \\
& \frac{D C}{D A}=\frac{A M}{M P} . \quad \therefore \frac{q a}{b}=\frac{x-a}{y} . \\
& \frac{A^{\prime} O}{O N}=\frac{A^{\prime} M}{M P} . \quad \therefore \frac{a}{q b}=\frac{x+a}{y} .
\end{aligned}
$$

Multiplying these two equations together member for member, we have $\frac{a^{2}}{b^{2}}=\frac{x^{2}-a^{2}}{y^{2}}$;

whence $a^{2} y^{2}-b^{2} x^{2}=-a^{2} b^{2}$, the equation of the $x$ hyperbola.

c) For the parabola take $O$ in Fig. 28 for the origin and $O X$ and $O Y$ for axes. Draw $A M$ and $\|$ to $A M$, right lines $N E$. Through the pts. $N$ draw lines $\|$ to $O X$; join pts. $E$ with $O$. The intersections $P$ are pts. of the parabola passing through $O, C, D$, and having $O X$ for a diameter and $O Y$ for a tangent at the vertex $O$.

Let $O A=b, O M=a, F P=q b, A E=q a ; \frac{F P}{O F}=\frac{O A}{A E}$.

Let $F P=y$ and $O F=x$; then $\frac{y}{x}=\frac{b}{q a}$, also, $y=q b$;

by multiplication $y^{2}=\frac{b^{2}}{a} x$, the equation of the parabola.

Рroв. 172. a) Let Fig. 29 represent a circle and circumscribed square: we wish to find pairs of pts. in the square which determine rectilinear tangents to the circle. Let a tangent with the pt. of tangency $k$ cut $a b$ and $a d$ in the pts. $p$ and $q$. 
Let $\quad u p=x, \quad q a=y$.

In the rt. $\triangle a p q$ we have

$$
\begin{aligned}
p a & =r-x \quad(r=\text { radius }), p q=p k+k q=x+r-y, \\
\overline{p q}^{2} & =\overline{p a}^{2}+\overline{q a}^{2} \text { or }=(x+r-y)^{2}=(r-x)^{2}+y^{2} . \\
\therefore y & =\frac{2 r x}{r+x} .
\end{aligned}
$$

Draw the chord $s u$; through $p$ draw the parallel to $u v$, cutting $s u$ in $r$; then draw $b r$ which cuts $a d$ in some pt. $q^{\prime}$ whose distance from $a$ we will designate by $y^{\prime}$. . . Then because

$$
b p: p r:: a b: a q^{\prime}
$$

or

$$
r+x: x: 2 r:: y^{\prime},
$$

we have $y^{\prime}=\frac{2 r x}{r+x} . \quad \therefore q$ falls upon $q^{\prime}$.

For the pt. of tangency, $k, p k=p u$, also $m p$ and $v k$ are $\perp$ to $u k$ and $\therefore \|, \therefore$ the intersection, $g$, of $v k$ and $m s$ lies upon $p r$. The parallel projection of a circle is an ellipse, while parallels will remain parallel, tangents will remain tangents, etc. Therefore the parallel projection of Fig. 29 will give the construction in the upper left-hand quadrant of Fig. 26 for an ellipse determined as the envelope of a system of tangents.

b) If a $\Delta$ of constant area be cut off by a moving line from two fixed intersecting lines, we know from Analytic Geometry that the envelope of the moving line is an hyperbola and that the middle pt. of the line is in every position the pt. of tangency. 'Taking the intersecting lines as axes, the inclucled angle as $\phi$, $K$ as the constant area of the $\Delta$ and $x$ and $y$ as the intercepts upon the axes, we have for the middle pt. of the moving line $\frac{1}{2} x \cdot \frac{1}{2} y \cdot \sin \phi=2 k . \quad \therefore x y=$ constant, the equation of an hyperbola referred to its asymptotes.

c) If two intersecting lines are divided into equal parts the pts. on one line beginning at the pt. of intersection being numbered from 1 to $n$, on the other from $n$ to 1 , and like numbers are joined by right lines, there will be formed the enveloping tangents of a parabola. 'The proof is from Projective Geometry. 
Prob. 173. When the principal axes, $A A^{\prime}, B B^{\prime}$, are given, and circles are described about them as diameters, a pt. upon the ellipse may be found as follows: Draw any line from the common centre intersecting both circles. From the outer pt. of intersection let fall a $\perp$ to the major axis, from the inner a $\perp$ to the minor axis. The pt. of intersection of the $\downarrow$ s is the rq. pt. For construction, see Fig. 30. When conjugate diameters are given, the method by circles is shown in Fig. 31.

Prob. 174. The construction of this problem is shown in Fig. 32. It is from Parallel Projection, and is known as the Shadow Method. Let $\alpha \alpha^{\prime}$ and $\beta \beta^{\prime}$ be the gn. conjugate axes. Throngh $\beta$ draw $E \|$ to $a \alpha^{\prime}$. $\quad E$ must be tangent to the ellipse whose axes are required and is taken as an axis of affinity. From $\beta$ erect $\beta c \perp$ to $E$ and $=\frac{1}{2} \alpha \alpha^{\prime}=\alpha o$. With $c$ as centre and $c \beta$ as radius, describe the circle $K$. The ellipse $K_{1}$ is the $\|$ pr. of $K$. All pairs of diameters $\perp$ to each other in $K$ will be projected as conjugate diameters in $K_{1}$. The pair of $\perp$ diameters of $K$ which remain $\perp$ in $K_{1}$, are the principal diameters of the ellipse. Pass through $o$ and $c$ a circle, $K^{\prime}$, with centre in $E$. The $\perp$ diameters $a a^{\prime}, b b^{\prime}$, of $K$ remain $\perp$ in $K_{\mathrm{t}}$ and are in projection the principal diameters $A A^{\prime}, B B^{\prime}$, sought.

Prob. 175. It is a fundamental proposition in transversals that if a right line intersect the three sides $A B, B C, C A$, of a $\triangle A B C$, or those sides produced in pts. $P_{1}, P_{2}, P_{3}$, respectively, then the product of three non-adjacent segments equals the product of the other three; or

$$
\frac{A P_{1} \cdot B P_{2} \cdot C P_{3}}{B P_{1} \cdot C P_{2} \cdot A P_{3}}=1
$$

and conversely, when the above relation holds, $P_{1}, P_{2}, P_{3}$, are in one right line. Starting from this proposition, we shall prove Pascal's proposition applying to his so-called Hexagrammatica Mystica. The proposition is this: if six pts. lie on the circumference of a circle and an inscribed convex or re-entrant hexagon be formed with these pts. as vertices, then the opposite sides, or sides produced, meet in three points lying upon a right 
line which we shall call the axis. In the $\triangle L M N$ of Fig. 33, let $A P, F Q, C R$, be taken successively as transversals.

We have

$\frac{L A \cdot M B \cdot N P}{A M \cdot B N \cdot L P}=1, \quad \frac{L F \cdot M Q \cdot N E}{M F \cdot N Q \cdot L E}=1, \quad \frac{\bar{L} R \cdot M C \cdot N D}{M R \cdot N C \cdot L D}=1$.

Also since $L D, L A$, etc. are secants,

$$
\frac{L E \cdot L D}{L F \cdot L A}=1, \quad \frac{M A \cdot M F}{M B \cdot M C}=1, \quad \frac{N C \cdot N B}{N D \cdot N E}=1 .
$$

Multiplying these six equations together we have

$$
\frac{N P \cdot M Q \cdot L R}{L P \cdot N Q \cdot M R}=1 \text {. }
$$

By the converse of the proposition regarding transversals, $P, Q, R$, must be in the same right line. Since the ellipse, hyperbola and parabola are formed by the central projection of the circle and since in such pr. rt. lines, tangents, pts. of intersection and pts. of tangency in the projected figure remain the same in the projection, it follows that the demonstration gn. applies to the curves of the second degree.

If five pts., $A, B, C, D, E$, are gn. in one plane, any number of lines $A F$ may be drawn, and the corresponding pts. $F$ may be found by using in each case the corresponding axis. Therefore, when five pts. are gn. in a plane, one conic section can always be found containing them and only one. An inscribed pentagon may be regarded as an inscribed hexagon in which one pair of adjacent vertices are consecutive; an - inscribed quadrilateral, one in which two pairs are so related; an inscribed triangle, one in which three pairs coincide. In the last three cases a pair of consecutive vertices are not fully gn. unless the direction of the line joining them is gn. This line is tangent to the conic section in which the consecutive vertices lie. If the five pts. assumed form a convex figure, the conic section containing them will be an ellipse, as in $a$ ) of this Prob. If the five pts. assumed form a re-entrant figure, the section will be an hyperbola, as in $a$ ) of 176 . If three pts. be 
assumed and a direction leading at infinity to two other pts., and if the latter are consecutive with the line joining them, the infinite right line of the plane, then the section will be a parabola, as in 177 .

The proposition of Brianchon is derived from that of Pascal by the application of the principle of pole and polar. It is as follows: If a hexagon be circumscribed to any one of the conic sections, the three diagonals joining opposite vertices will pass through the same pt. In Pascal's hexagram it is convenient to arrange the symbols for the lines in a row and connect them as rq. If $A, B, C, D, E, F$, be the vertices, then $A B, B C, C D$, $D E, E F, F A$, will be the sides, and $A B \cdot D E, B C \cdot E F, C D \cdot F A$, will be the three collinear intersections.

In Brianchon's hexagon $a, b, c, d, e, f$, will be the vertices and $a d, b e, c f$, the lines which intersect in a pt. It is evident that in the former, when five vertices are gn. any number of sixth vertices belonging to the same conic section can be found. In the latter, five tangents being gn. any sixth tangent can be found.

Two pts. each of $a$ ) and $b$ ) are constructed in Figs. 34 and 35. In Fig. 34, the axis always passes through $P$. In finding $f$ it also passes through the intersection of $b c$ and the assumed direction ef. Similarly for $e f^{\prime}$. In Fig. $35, A, B$, etc., denote the sides of the gn. pentagon; the intersection of $A$ and $B$ is denoted by $A \cdot B$, of $B$ and $C$, by $B \cdot C$, etc. Then the order for finding any number of tangents, $F$, is

$$
A \cdot B, B_{\mathrm{L}} C, C \cdot \overline{\bar{D}, D} \cdot E, E, F, F \cdot A .
$$

Two tangents, $F$, are determined in Fig. 35. Determine the principal axis by 174 .

Prob. 177. Two pts. of the rq. parabola are constructed in Fig. 36. $a b$ is produced to intersect at $\infty$ with the infinite right line $d e$; the latter is the direction to $a b$. The line $f^{\prime} e$ is assumed. Its intersection with $b c$ determines one pt. of $E$, $E$ being drawn to the intersection of $a b$ with de must be $\|$ to $a b$.

РRов. 179. Use as an auxiliary line the element upon which $a$ is found. 
Рroв. 181. Use the plane $\perp$ to $o o_{1}$ at $o$ as an auxiliary surface and its traces as axes of affinity between the elliptical projections of the base and its circular developments.

Рвов. 183. The development is theoretically effected by opening the cone along some element and rolling the surface out into a plane. It is practically done by dividing the base into any number of equal parts. The true lengths of the elements passing through these pts. of division are found as nearly as practicable and the true length of the equal arcs. These constituent lines combined in the proper order form triangles, which, placed adjacent in a plane in the same relation in which they stand in the surface, are the development of the latter. A continuous curve passed through the non-concurrent ends of the developed elements is the developed base. This work requires the rectification of the circle. Three and one-seventh times the diameter is 0.0013 of the circumference too much. Three times the diameter increased by one-fifth the chord of a quadrant is 0.0003 of the circumference too small. Both of these errors are smaller than those that ordinarily arise from the use of instruments.

When the base of the cone is a curve not easily rectified, it is usually accurate enough for descriptive purposes to take divisions of the base so small that the arcs shall not sensibly differ from their chords. Occasionally the development is effected by using the intersection of the cone with a sphere whose centre is at the vertex. The developed intersection is then the arc of a circle.

Proв. 185. By Analytic Geometry it is proven that the intersection of a plane with a cone of the second order is

a) an ellipse, when it cuts all the elements ;

b) a parabola, when it is parallel to one and only one element;

c) an hyperbola, when it is parallel to two elements.

Take $t^{\prime} T t^{\prime \prime}$ so as to give $\left.a\right), b$ ), and $c$ ), successively.

Prob. 187. Similar to 101.

Prob. 188. Similar to 100 , 
Рrob. 189. Revolve the line upon its $H$-pr. into $H$ together with the small circle cut out from the sphere by its $H$ projecting plane.

Рroв. 190. In elementary Geometry it is shown that the line cut out from the plane of the base of the cone by the tangent plane - in other words the $V$ trace of the latter as here given - must be tangent to the base of the cone at the foot of the element containing $p . \quad T t^{\prime \prime}$ and the pt. where a line from $S$ or any pt. of the element of tangency and $\|$ to $T t^{\prime \prime}$ pierces $H$, will determine $t^{\prime} T$.

РRов. 192. Draw an auxiliary line through $S$ and $p$ and find its $h . t^{\prime} T$ must pass through $h$ and be tangent to the base of the cone. In general, there are two solutions for cones of the second order.

РRoв. 193. The auxiliary line is drawn through $p \|$ to the elements of the cylinder. Otherwise as in 192.

Рroв. 196. Pass an auxiliary line through $S \|$ to the direction $m n$, which is a gn. line.

Prob. 198. Construct an auxiliary cone with vertex at any pt., $p$, and with elements making with $H$ the $\Varangle \epsilon$. Through $p$ pass a line $\|$ to the elements of the cylinder. The tangent plane upon the auxiliary cone determined by this line will be II to the plane rq.

Рвов. 199. Construct an auxiliary cone of revolution with its vertex at the vertex of the cone and its elements making the $\Varangle \in$ with $H$. The common tangent plane will be the plane rq.

Prob. 200. The helix is defined in Part III., Art. 27, and is constructed in Fig. 37.

The developable or tangential surface is defined in Part III., Art. 46, and constructed upon the helix of Fig. 37.

When the helix with axis $\perp$ to $H$, forms the directrix as in this case, the base of the surface in $H$ is the involute of the circle forming the $H$-pr. of the helix. If a rt. $\triangle$ be cut from paper and wound about a cylinder with one of its legs \| to the axis of the latter, the hypotenuse will be very nearly a 
helix. Unwind the paper, keeping the unwound portion plane. The hypotenuse will approximately describe a developable surface and the vertex at the lower acute angle the involute base. The tangent plane is drawn as in the cone.

Pros. 201. Take the axis of the helical directrix $\perp$ to $H$. With any pt. of $a b$ as a vertex, describe an auxiliary cone of revolution whose elements make with $H$ the same $\Varangle$ as the elements of the gn. surface. The plane containing $a b$ and tangent to the anxiliary cone will be $\|$ to $t^{\prime} T l^{\prime \prime}$.

Prob. 202. Take auxiliary planes as in 103.

Рвов. 203. The problem is similar to 96 . The auxiliary planes will be $\|$ to a plane determined by two intersecting lines; one $\|$ to the elements of the cylinder, the other to the edges of the prism.

Рвов. 205. Auxiliary planes cutting rectilinear elements from both surfaces must contain the line passed through $S$ $\|$ to the elements of $K$. In other words, they must pass through the $v$ and $h$ of this line. Especial attention should be given to the auxiliary planes whose traces are tangent to the bases of one or both of the surfaces, for such planes contain elements which are tangent to the line of intersection. The solution is similar to Fig. 21.

Рвов. 208. $t^{\prime} T t^{\prime \prime}$ will be $\perp$ to the radius of tangency at $p$.

Рвов. 209. Construct auxiliary cones of revolution tangent to the sphere and with vertices in $v$ and $h$ of $a b$. The planes of the bases of these cones will be the corresponding projecting planes of the chord of the sphere, connecting the two possible pts. of tangency. By revolving either projecting plane into its corresponding plane of pr. and with it the small circle cut by it from the sphere, the pts. of tangency are found.

Prob. 210. Pass a plane through $c \perp$ to $a b$ and determine the pt. cut from $a b$. Develop into either $H$ or $V$ this pt. and the great circle cut from the sphere. The tangent from pt. to circle is the developed position of a tangent intersecting $a b$ and with it determining $t^{\prime} T t^{\prime \prime}$. Two solutions.

Prob. 211. An hyperboloid of revolution of one nappe or 
sheet is generated by the revolution of an hyperbola about its imaginary axis. It is also generated by the revolution of one right line about another to which it is windschief and with respect to which its relation is constant. To prove the surface thus generated is an hyperboloid of one sheet, it is only necessary to prove that its meridian section is an hyperbola whose imaginary axis is the axis of revolution.

In Fig. 38 , let $v$ be any pt. in the meridian line, $t^{\prime \prime} m^{\prime \prime} v^{\prime \prime}$. Let $a b$ be an element $\|$ to $V$. Let $o^{\prime} m^{\prime}=o^{\prime} d^{\prime}=d^{\prime \prime} m^{\prime \prime}=a=$ the radius of the circle of the gorge. In the rt. $\Delta d^{\prime \prime} m^{\prime \prime} c^{\prime \prime}$, let $m^{\prime \prime} c^{\prime \prime}$ $=b$. Let $r^{\prime} v^{\prime}=x=r^{\prime} s^{\prime}=r^{\prime \prime} v^{\prime \prime}$, and $d^{\prime \prime} r^{\prime \prime}=y$.

In the rt. $\triangle r^{\prime} d^{\prime} s^{\prime}$

$$
{\overline{r^{\prime} s^{\prime}}}^{2}={\overline{r^{\prime} d^{\prime}}}^{2}+{\overline{d^{\prime} s^{\prime}}}^{2} . \quad \therefore x^{2}=a^{2}+\overline{d^{\prime} s^{2}} ;
$$

but

$$
\begin{gathered}
d^{\prime} s^{\prime}=r^{\prime \prime} s^{\prime \prime}, \text { and } \frac{r^{\prime \prime} s^{\prime \prime}}{r^{\prime \prime} d^{\prime \prime}}=\frac{d^{\prime \prime} m^{\prime \prime}}{m^{\prime \prime} c^{\prime \prime}} \text {, or } d^{\prime} s^{\prime}=\frac{a}{b} y . \\
\therefore x^{2}=a^{2}+\frac{a^{2}}{b^{2}} y^{2} \text {, or } a^{2} y^{2}-b^{2} x^{2}=-a^{2} b^{2},
\end{gathered}
$$

the equation of the $x$ hyperbola. Read Part III., Arts. 53-62.

The revolution of the common $\perp$ generates the smallest parallel of the surface. This parallel is called the circle of the gorge.

A right line intersecting the generatrix at the foot of the common $\perp$ from generatrix to axis and making with the latter the same angle in space, will evidently generate the same surface, since every pt. of the new generatrix will be at the same distance from the axis as its homologous pt. in the old generatrix. Therefore through any pt. of the surface there will pass two rectilinear elements, one from each generation, and these determine the tangent plane at that point. It is also easily seen that an element of one generation cuts every element of the other.

Let the hyperboloid be cut by two planes $\perp$ to the axis and at equal distances above and below the plane of the gorge. If the axis is $\perp$ to $H$, an element joins pts. in these bases whose $H$-prs. are separated by an arc greater than zero and less than 
$180^{\circ}$. The tangents at these two pts. are therefore not $\|$, and, since they do not intersect, they are windschief. An element and its successive element, therefore, are lines connecting different pts. of two windschief lines and must themselves, therefore, be windschief. Hence the surface itself is warped. See Part III., Art. 44, 3.

Pros. 212. The tangent plane is determined most readily by two tangents through $p$, one to the meridian section, the other to the parallel through the gn. pt.

Prob. 214. If with the given line as generatrix and the axis of the given solid as axis, an hyperboloid of revolution of one sheet be formed, the tangent plane will be tangent to this auxiliary surface at some pt. of the given line ; for a plane containing an element of a warped surface must be tangent to that surface at some pt. of the element. See Part III., Art. 66.

Also, the meridian plane passed through the pt. of tangency of the given solid must cut ont a common tangent from the common tangent plane. If, then, we lay in $V$ the common tangent upon the bounding lines of the two surfaces of revolution, and determine the pt. of tangency upon the hyperboloid, that pt. will give the parallel circle containing the pt. of the given line where the latter is cut by the common meridional tangent. This tangent, with the given line, determines the rq. plane.

Prob. 215. Pass a system of planes through $p$ and the ellipsoid and to each section determine the tangent line and the pts. of tangency. The former will be the elements of the rq. cone; the latter, pts. in the rq. curve of contact. This curve is plane and of the $2 d$ order.

Рвов. 216. Use the projecting planes of the cylindrical elements as auxiliary surfaces. In general, they cut right lines from the cylinder and small circles from the sphere whose mutual intersection is determined by revolution.

Рвов. 217. For auxiliary surfaces take spheres with their common centre at the intersection of the axes.

Prob. 218. Assume an axis $\perp$ to $I$ whose $H$-pr. is the middle pt. of an ellipse assumed as the basal section and the 
projection of the upper base. Lay off at will in $V$, along the axis from $G$, any distance as the altitude of the bounded solid. The plane of the ellipse of the gorge bisects this distance. The $H$-pr. of the gorge may be assumed, in which case it is concentric with the base and similar to it. The hyperboloid of revolution is best represented by dividing the circular base into any number of equal parts and drawing elements from the pts. of division. In the general hyperboloid under discussion, the foot points of elements corresponding to these may be found $b y$ describing a circle about the major axis, dividing this circle into any number of equal parts and projecting the pts. of division upon the ellipse by lines $\perp$ to the major axis. This construction is seen to resemble the method of Fig. 30 and is in reality dependent upon the orthographical projection of a circle whose plane is oblique to the plane of projection. A pt. in the base of the asymptote cone is found by passing through the axis a plane parallel to any element and projecting its foot pt. upon this plane. Read also Part III., Arts. 63-74.

Pros. 219. Without entering further into the discussion of this surface, it may simply be stated that it is one of the limiting cases of the hyperboloid of one nappe and, like that surface, bas a double system of generation.

To represent the surface symmetrically, divide portions of two windschief lines into the same number of equal parts and join homologous pts. Any two elements of one generation may be taken as the directrices of the second with which the operation given above may be repeated, as. shown in Fig. 39. Various beautiful projections of this interesting surface can be easily contrived.

The two rectilinear elements passing through any pt. of a surface determine the tangent plane at that pt.

Рroв. 220. Through $p$ and $c d$ pass a cone intersecting the projecting cylinder of $e f$ in a curve, $x y$, whose actual intersection with ef determines a second pt., $q$, in the rq. element. The construction is given in Fig. 40.

Prob. 221. For case $a$ ), construct a series of lines in the 
plane directer. Through $p$ pass a cone of rays $\|$ to this series and find a second pt. in the rq. element, as in 220.

For case $b$ ), pass through $a b$ a cylinder of rays, each of which is parallel to $m n$. Find a second pt. as in 230 .

Prob. 222. Construct the helical directrix with axis $\perp$ to $H$. Divide the circular base into any number of equal parts. Draw one of the clements lying $\|$ to $V$ at the gn. $\Varangle$ in $V$ with $a^{\prime \prime} b^{\prime \prime}$, and intersecting the latter in $S^{\prime \prime}$. Divide the portion of the axis in $V$ above and below $S^{\prime \prime}$ corresponding to one spire of the helix into the same number of equal parts as the circular base. Connect the successive pts. in the helix, above and below the element already drawn, with the corresponding pts. of the axis.

Assume a pt. in the surface by assuming one of its prs. and finding the other by constructing the rectilinear element upon which it lies.

Рroв. 223. To pass a plane tangent to a helicoid at any pt. of its surface, we construct the subordinate helix upon which the pt. lies and the right line tangent to this helix at the gn. pt.

The constructed tangent and the rectilinear element passing through the pt. determine the plane sought.

Рвов. 225. The auxiliary surfaces most convenient for application are planes $\perp$ to the axis of the hyperboloid. The principal axis of the section is the intersection of $t^{\prime} T t^{\prime \prime}$ with that meridian plane which is $\perp$ to it. 


\section{PART III.}

\section{SUMMARY OF PRINCIPLES AND DEFINITIONS.}

\section{Section I. \\ Projections.}

1. Descriptive Geometry. Descriptive (darstellende, beschreibende) Geometry is the science and art of the methods by which the form and position of geometrical solids are represented by drawings and by which all constructions rq. in space can be solved with the help of such elements as can be represented in a plane.

2. Shading and Perspective. Descriptive Geometry includes Shading and Perspective and is, therefore, the foundation of the art of Drawing.

3. The representation of a solid may be effected in general in two ways, giving rise to Central Projection and Parallel Projection.

4. Central Projection. The Central Projection of a figure is the intersection of a plane and a pencil of rays.

The plane is the Plane of Projection, or the Picture Plane. The pencil of rays is composed of straight lines, each passing through the fixed or central point - called the Point of Sight-, a point of the figure and a point of the picture plane.

5. Parallel Projection. The Parallel Projection of a solid is the intersection of a plane and a system of parallel lines : it is, in fact, central projection with the point of sight at infinity.

The plane is the Plane of Projection; the parallel lines, Projecting Lines. All the projecting lines of a continuous figure in space form a Projecting Cylinder. For the right line in general and for special positions of plane figures, the projecting cylinder becomes a Projecting Plane. Parallel projection is Oblique or Orthogonal.

6. Oblique Parallel Projection. In oblique parallel projection the projecting lines are oblique to the plane of projection. 
7. Orthogonal Parallel Projection. In orthogonal or orthographic parallel projection the projecting lines are perpendicular to the plane of projection.

8. In this hand-book, whenever the projection of a figure is mentioned, the orthographic is meant, unless otherwise specified, and Descriptive Geometry will be taken to mean the science and art of orthographic progression.

9. Planes of Projection. In orthographic projection usually two principal planes of projection are taken at right angles to cach other, one the horizontal, $H$; the other the vertical, $V$. To these may be added a third, $P$, perpendicular to both. Except in special positions of the figure to be projected, it is fully determined without the aid of $P$.

10. Ground Line. The intersection of $H$ and $V$ is the ground line, $G$; of $V$ and $P$, the $G_{2}$; of $P$ and $H, G_{3}$.

11. Revolutions. By revolution through an angle of $90^{\circ}$ about $G, H$ and $V$ are made coincident. To effect this transformation, which is the fundamental one of Descriptive Geometry, we may suppose the part of $V$ above $G$ revolves backwards, the part below $G$ forwards through $90^{\circ}$, or that the back part of $H$ revolves upwards, the fore part downwards, through the same angle. For the sake of uniformity the latter change will be always understood, unless otherwise specified. When $H$ and $V$ have been made coincident, the part of the composite plane above $G$ represents upper $V$ and back $H$; the part below $G$, front $H$ and lower $V$. Similarly $P$ is revolved through $90^{\circ}$ upon $G_{2}$ and it will be uniformly assumed that the fore part of $P$ is superposed upon the right-hand part of $V$.

\section{Section II.}

Point, Line and Plane.

12. Point. A point is completely determined by its two projections. The projections of a point lie in the same line $\perp$ to $G$. 
13. Right Line. The projections of a right line are also right lines. In general, a line is completely determined by its first two projections, except when it lies in a plane $\perp$ to $G$.

14. Traces of a Line. The points in which a line pierces $H$, $V$ and $P$ are its traces, $h, v, p$.

Note. Statements will now be confined to the first two projections. The student will readily make and prove similar statements for the third projection.

15. The $h$ and $v$ of a line are in its $H$-pr. and $V$-pr., respectively, and are their own projections of the same name. Also the $V$-pr. of $h$ and the $H$-pr. of $v$ are in $G$. If, therefore, from the point in which the $V$-pr. of the line cuts $G$ a $\perp$ to $G$ be drawn in $H$, its intersection with the $H$-pr. of the line is $h$ : similarly for $v$.

16. Angle of Inclination. The angle of inclination of a line or plane is the angle it makes with a plane of projection.

17. Parallel Right Lines. If two right lines are $\|$, their like-named projections are $\|$.

18. Plane, how represented. The position of a plane is in general represented by its intersections with $H$ and $V$.

These intersections are called the traces of the plane and must always meet $G$ in the same point.

19. A plane is determined by three points not in the same right line, by a line and point without it, by two intersecting lines and by two parallel lines.

20. Point in Plane. If a point lies in a plane, it lies in any line of that plane whose projections pass through the projections of the point.

21. Line in Plane. If a line lies in a plane, its $h$ and $v$ lie respectively in the $H$ and $V$ traces of the plane.

22. Plane perpendicular to $\mathrm{H}$ or $\mathrm{V}$. If a plane is perpendicular to $V$ its $H$ trace is perpendicular to $G$ and vice versa.

23. Line perpendicular to Plane. If a line is $\perp$ to a plane 
its projections are $\perp$ to the like-named traces of the plane and conversely.

24. Two Parallel Planes. If two planes are Il, their homologous traces are $\|$. The converse is true, except when the planes are \| to $G$, then their third traces must be il to prove the planes $\|$.

\section{Section III. \\ Line in General.}

25. Line, how Generated. A line is generated, $a$ ) by a moving point, $b$ ) as the envelope of a moving right line.

26. Plane Curve. A line is plane when four consecutive points always remain in the same plane.

27. Space Curve. A line is a space curve when four consecutive points do not generally remain in the same plane.

28. Plane Curves.

1. Algebraical. The locus of a rational algebraical equation, in general of two unknown quantities.

a) Order. Number of times intersected by a right line. First Order. Right line (a circle of infinite radius and thus a curve).

Second Order. Ellipse, parabola, hyperbola.

b) Class. Number of tangents that can be drawn to it from any point in its plane.

First Class. Point.

Second Class. Ellipse, parabola, hyperbola.

2. Transcendental. The locus of a transcendental equation; either trigonometrical, circular, logarithmic, or exponential.

29. Higher Plane Curves. All plane transcendental curves and plane curves of a higher order or class than the second, are called higher plane curves.

30. A transcendental curve may in general be intersected by a right line in an unlimited number of points.

Examples of the more common and useful transcendental 
curves are the curves of the trigonometric functions, as the sine, tangent; the rolling curves, as the cycloid, epicycloid, hypocycloid; the spirals, as the involute of a circle, spiral of Archimedes, logarithmic spiral.

31. Tangent. A tangent to a curve is a right line passing through any two of its consecutive points. A curve is always convex towards a tangent at the point of contact.

32. Normal. A normal to a curve is a right line drawn $\perp$ to a tangent at the point of tangency. In plane curves the normal is taken in the plane of the curve.

33. Axis and Vertex. If a normal divides a curve into two symmetrical parts, it is an axis, and its point of intersection with the curve is a vertex.

34. Diameter. If we draw over a plane curve a group of $\|$ chords and join their centres successively with a line, the latter is a straight or curved diameter.

35. Osculating Circle and Radius of Curvature. The circle which passes through any three consecutive points of a curve is an osculating circle, and its radius is the radius of curvature for the curve at the middle of the three points. The radius is usually represented by $\rho$.

36. Space Curves. The number of space curves is unlimited, but the two of special interest are the helix and the spherical epicycloid.

37. Helix. The Helix is generated by a point revolving about a fixed right line called its axis. The generating point remains at a constant distance from the latter and bas a velocity of translation in its direction in a constant ratio to its angular velocity about it.

38. Spherical Epicycloid. The spherical epicycloid is generated by a point in an element of one cone of revolution rolling upon another, both cones having the same vertex and always one and but one element in common. 


\section{Section IV. \\ Surfaces in General.}

39. Surface. A surface is the locus of the different positions of a line, called the generatrix, or it is the envelope of the different positions of other surfaces. The law for the motion must state whether the generating figure remains of unchanging form or not.

40. Directrix, Directer. Fixed lines along which the generatrix glides, and fixed surfaces against which it assumes definite positions, are called directrices and directers.

41. Kinds of Surfaces. Since surfaces in general involve the idea of three dimensions in space, Analytic Geometry uses equations involving three variables to designate them and divides them into algebraical and transcendental in the same way that it divides lines.

42. Algebraical surfaces are divided into order's and classes!

Nth Order. A surface of the $n$th order is one that is cut by every plane in a line of the $n$th order. The surface of the first order is the plane.

43. Families of Surfaces. Irrespective of these divisions, surfaces are brought together in families, members of the same family having something in common in their generation.

The majority of surfaces applied in the arts belong to two such groups, the first family has the simplest line, - the right line,-for generatrix; the second is formed by the simplest motion of any line, - revolution about a fixed axis. These groups are called ruled surfaces and surfaces of revolution. Several special forms belong to both families.

44. Ruled Surface. A ruled surface is the locus of a right line moving in conformity with a given law.

1 The student is referred to Analytic Geometry of three dimensions, for the discussion of classes of surfaces. 
There are three subdivisions:

1. Plane.

2. Developable surfaces, or such as have every two consecutive positions of the generatrix in the same plane.

3. Warped surfaces, or such as have every two successive positions of the generatrix windschief.

Note. Any two right lines crossed in space, but not intersecting, are called windschief.

45. Double Curved Surface. Any geometrical surface not belonging to the ruled surfaces belongs to the double curved surfaces.

\section{Section $\mathrm{V}$.}

\section{Developable Surfaces.}

46. Developable Surface. A developable surface is also the envelope of a plane which moves in accordance with some given law, but the simplest generation is to cause a right line to move constantly tangent to any space curve. It might, therefore, be called a tangential surface. A plane element of this surface will be the portion of a plane included between two successive and intersecting positions of the generatrix. Therefore the surface can be developed into a plane by enlarging the dihedral angle between all successive plane elements to $180^{\circ}$. at every stage keeping the plane elements already developed fixed, while the remaining elements are consecutively added thereto.

47. Developable Surface with Helical Directrix. An example of the developable surface is that with a helical directrix, in which the space curve along which the generatrix glides is, as the name indicates, the helix.

48. A simple developable surface arises when the space curve directing the generatrix is reduced to a fixed point. In this case the motion of the generatrix is to be restricted by another condition. 
49. Cone. A cone is the locus of a right line which always contains a fixed point, $m$, while it passes successively through all the points of a given curved line or directrix. If the directrix is a plane curve, $m$ must not lie in the plane of the curve.

When the directrix is a curve of the second order, the cone is a surface of the second order.

50. Cylinder. If the fixed point $m$ lies in infinity, the sides of the surface are parallel and the cone becomes a cylinder whose order is determined in the same way as that of the cone.

51. Tangent Plane. A tangent plane to a surface at a given point is the plane which contains all the tangent lines to the surface at that point. Any two of these lines are sufficient to determine the plane. In general, the plane tangent to a developable surface at a given point upon it, is most readily determined by the element through the point of tangency and the line tangent to the base of the surface at the point in which the element of tangency pierces the plane of the base.

52. Shortest Path. The shortest path upon a developable surface between two points in that surface, is the right line joining those points when the surface has been developed. On the cylinder of revolution this line becomes identical with the helix and the loxodrome.

\section{Section VI. \\ Surfaces of Revolution.}

Note. Before taking up warped surfaces, we will consider surfaces of revolution, as one warped surface, the hyperboloid of one sheet, in the form most applied in the arts, is a surface of revolution and can be most conveniently treated as such.

53. Surface of Revolution. A surface of revolution is the locus of any line which remains unchanged in form and in position with reference to a right line about which it revolves without a motion of translation.

54. Axis. The fixed line is called the axis of revolution, or simply the axis. 
55. Parallels. Every plane intersecting the surface and perpendicular to the axis cuts out a circle. All such circles are called parallels.

56. Meridian. Every plane passed through the axis cuts out a curve called a meridian. All meridians of the same surface of revolution are equal and symmetrical with respect to the axis. The meridian curve of a surface of revolution is also called its profile.

57. Equator. When a plane of symmetry for a meridian curve can be found perpendicular to the axis, the parallel cut by it is called the equator of the surface.

58. Representation of Surfaces of Revolution. By the definition of surface of revolution, we know two systems of lines, and these serve to represent the surface. The representation is accomplished most simply by placing the axis perpendicular to $H$, when the boundary of the $V$ projection will be a meridian section, while the $H$ projection will be either the parallel cut out by the $H$ plane, or the intersection with $H$ of the tangent horizontal projecting cylinder.

59. Circle of the Gorge. A parallel whose radins is smaller than that of any other, but greater than zero, is a circle of the gorge.

60. According as the meridian is a transcendental or algebraical line of the $n$th order, so also is the surface of the same kind and order.

61. Surfaces of revolution may be divided into orders, as follows :-

First Order. The meridian is a right line $\perp$ to the axis of revolution, - plane.

Second Order. The meridian is composed of :

a) Two lines parallel to and equally distant from the axis, - cylinder.

b) Two lines intersecting the axis and equally inclined to it, - cone.

c) A circle with centre in the axis, - sphere. 
d) An ellipse whose minor or major axis lies in the axis of revolution, - the oblate or prolate spheroid.

e) A parabola with axis as axis of revolution, paraboloid.

f) An hyperbola whose real axis is the axis of revolution, - liyperboloid of double sheet.

g) An hyperbola whose imaginary axis is the axis of revolution, - hyperboloid of single sheet. $g$ ) may also be generated by revolving one right line about another to which it lies windschief.

Higher Orders. Other kinds of revolution of lines of the second order, give surfaces of the fourth order often applied in the arts for vases, light-house towers, \&c. The conchoid of Nicomedes, the oval, the logarithmic curve, the cosine curve and allied forms, the cycloid, etc., generate surfaces of revolution much used in the arts and which are algebraical or transcendental according as the meridian curves are the one or the other.

62. Tangent Plane. When the point of tangency on a surface of revolution is given, the tangent plane is generally determined most readily by the tangent at the given point to the parallel and the tangent through the same point to the meridian.

This method is simplified in the cone and cylinder, while in the hyperboloid of revolution of one sheet, a rectilinear element passing through the point of tangency is taken from each of the two systems of generation.

\section{Section VII.}

\section{Warped Surfaces.}

63. Warped Surface, A warped surface is a ruled surface in which any two consecutive rectilinear elements are windschief. Since no limit can be placed to the laws which shall 
govern the motion of a right line in space, none can be placed to the number of possible warped surfaces. In general, the generatrix will have directing points, lines, or surfaces which it shall intersect or intersect at a given angle; for example, simply touch or touch so as to cut a given system of lines at a given angle.

64. These laws can be variously expressed; as, for example, the generatrix shall always be at a given distance from a fixed point, means also that it shall always be tangent to a sphere of given radius.

65. The simplest law for the generation of warped surfaces is that the generatrix shall glide along three given lines, always intersecting all three. If every two consecutive elements intersect, the surface becomes a developable one; in general this is not so, and the surface is warped.

66. Property of a Plane containing an Element of a Warped Surface. Since each element of a warped surface is in general windschief with respect to every other, a plane containing one element will be pierced by every other element. These points of piercing together form a curve intersecting the given element. The tangent to this curve at the point where it intersects the given element, together with the element itself, forms two intersecting lines tangent to the surface at the same point. They therefore determine the plane which we have chosen, with the single condition that it shall contain an element, and make of it a tangent plane. Therefore, in general, it follows that any plane containing an element of a warped surface will be tangent to the surface at some point of the element.

67. Mutually Tangent Warped Surfaces. If two warped surfaces have an element in common and are tangent to each other at three points, $a, b, c$, of the same, then they are tangent along the entire element. For if we intersect the two surfaces at the given points by three planes, the latter must cut three linear directrices from each surface. Each pair of directrices must bave the point of tangency and its consecutive 
point in common, that is, $a a_{1}, b b_{1}, c c_{1}$; therefore these pairs of common points serve to determine two consecutive positions of the generatrix. It follows that a common warped surface element will be determined, and that a plane intersecting the surfaces at any other point than $a, b$, or $c$, of the common element, must cut out two consecutive points common to each surface.

68. Divisions of Warped Surfaces. A linear directrix can be a right line or a curved line (the latter a plane or space curve). There are, therefore, four kinds of warped surfaces, distinguished as follows; the directrices are:

1) Three right lines, - the hyperboloid of one sheet.

2) Two right lines and a curved line.

3) One right line and two curved lines.

4) Three curved lines.

69. Infinite Directrices. A right line can be one of the infinite right lines of space. In this case it determines with every point in space not contained in itself a plane, and all the planes determined by it and finite points of space are parallel.

If two infinite right lines, or two parallel right lines, are used as directrices, they will have an infinite point in common and the generatrix must always pass through this point. Therefore the surfaces formed with two such directrices and any kind of a line used as a third directrix, will be planes or cones (regarding the cylinder as a special form of the cone). If the two infinite directrices were curved they would have as many infinite points of intersection as the orders of the curves would allow; therefore, with a third directrix, there would now be formed a group of planes or cones.

70. But One Infinite Directrix. It follows that a warped surface can have but one infinite line as directrix, and since such a line gives rise to a series of parallel planes, we may take any one of these as a plane directer and every element will be parallel to this plane, while at the same time it intersects the other two directrices. 
71. Special Group of Warped Surfaces. There is thus formed a special group of warped surfaces, divided as follows :

The generatrix shall be always parallel to a given plane and shall cut

1) Two right lines, - the warped plane, or hyperbolic paraboloid.

2) One right line, one curved line, - the warped cone or conoid.

3) Two curved lines, - the warped cylinder or cylindroid.

72. Orders of Warped Surfaces. Among warped surfaces there are two of the second order, the hyperboloid of one sheet formed by a right line gliding upon three right lines, no one of which is an infinite right line of space; the hyperbolic paraboloid formed by a right line gliding upon three right lines, one of which is an infinite right line of space, or by a right line always parallel to a given plane moving along two other windschief right lines.

73. Higher Orders. All other warped surfaces are of higher orders, either transcendental or algebraic, according to the orders and kinds of the directrices and generatrices.

74. Screw Surface. Among the most important of those of higher order is the screw surface. It is generated by a right line intersecting at a constant angle and revolving about another right line, the point of intersection having a velocity of translation along the linear directrix in a constant ratio greater than zero to the angular velocity of rotation of the generatrix about it. When the angle of intersection is a right angle, the screw surface thus formed is also a warped cone or conoid, applied in spiral staircases, etc. 



\section{MATHEMATICS.}

Bowser's Academic Algebra. A complete treatise through the progressions, includ ing Permutations, Combinations, and the Binomial Theorem. Half leather. \$r.25.

Bowser's College Algebra. A complete treatise for colleges and scientific schools. Half leather. \$1.65.

Bowser's Plane and Solid Geometry. Combines the excellences of Euclid with those of the best modern writers. Half leather. $\$ x .35$.

Bowser's Plane Geometry. Half leather. $85 \mathrm{cts}$.

Bowser's Elements of Plane and Spherical Trigonometry. A brief course prepared especially for High Schools and Academies. Half leather. \$1.0.

Bowser's Treatise on Plane and Spherical Trigonometry. An advanced work which covers the entire course in higher institutions. Half leather. \$1.65.

Hanus's Geometry in the Grammar Schools. An essay, together with illustrative class exercises and an outline of the work for the last three years of the grammar school. 52 pages. $25 \mathrm{cts}$.

Hopkin's Plane Geometry. On the heuristic plan. Half leather. 85 cts.

Hunt's Concrete Geometry for Grammar Schools. The definitions and elementary concepts are to be taught concretely, by much measuring, by the making of models and diagrams by the pupil, as suggested by the text or by his own invention. roo pages. Boards. $30 \mathrm{cts}$.

Waldo's Descriptive Geometry. A large number of prob'ems systematically arranged and with suggestions. $90 \mathrm{cts}$.

The New Arithmetic. By 300 teachers. Little theory and much practice. Also an excellent review book. 230 pages. $75 \mathrm{cts}$.

For Arithmetics and other elementary work see our list of books in Number.

\section{C. HEATH \& CO., PUBLISHERS,}

BOSTON. NEW YORK. CHICAGO. 


\title{
ARITHMETIC.
}

Aids to Number. - First Series. Teachers' Edition.

Oral Work - One to ten. 25 cards with concise directions. By ANNA B. BAdeAm, Principal of Training School, Lewiston, Me., formerly of Rice Training School, Bosten. Retail price, 40 cents.

Aids to N (umber. - First Series. Putils' Edition.

Written work. - One to ten. Leatherette. Introduction price, 25 cents.

Aids to N Number. - Second Series. Teachers' Edition.

Oral Work. - Ten to One Hundred. With especial reference to multiples of numbers

from 1 to 10.32 cards with concise directions. Retail price, 40 cents.

Aids to Dembers. - Second Series. Pupils' Edition.

Written Work. - Ten to One Hundred. Leatherette. Introduction price, 25 cents.

The Child's eNumber Charts. ву Аnмa в. Вадеam.

Manilla card, $11 \times 14$ inches. Price, 5 cents each; $\$ 4.00$ per hundred.

Drill Cbarts. By C. P. Howland, Principal of Tabor Academy, Marion, Mass.

For rapid, middle-grade practice work on the Fundamental Rules of Arithmetic. Two cards, $8 \times 9$ inches. Price, 3 cents each; or $\$ 2.40$ per hundred.

Review Number Cards. By Erla M. Pirrce, of Providence, R. I.

For Second and Third Year Pupils. Cards, $7 \times 9$ inches. Price, 3 cents each; or $\$ 2.40$ per hundred.

Picture Problems. By Miss H. A. Luddington,

Principal of Training School, Pawtucket, R. I. ; formerly Teacher of Methods and Training Teacher in Primary Department of State Nornal School, New Britain, Conn., and Training Teacher in Cook County Normal Schonl, Normal Park, Ill. 7o colored cards, $4 \times 5$ inches, printed on both sides, arranged in 9 sets, 6 to ro cards in each set, with card of directions. Retail price, 65 cents.

\section{Mathematical Teacbing and its Modern Methods.}

By Truman Henry Safford, Ph. D., Professor of Astronomy, Williams College, Mass. Paper. 47 pages. Retail price, 25 cents.

\section{The New Aritbmetic.}

By 300 authors. Edited by Skymour Eaton, with Preface by T. H. Safrord, Pro fessor of Astronomy, Williams College, Mass. Introduction price, 75 cents.

\section{C. HEATH \& CO., Publishers,}

\author{
BOSTON, NEW YORK, AND CHICAGO.
}




\section{DRAWING AND MANUAL TRAINING.}

Johnson's Progressive Lessons in Needlework. Explains needlework from its rudiments and gives with illustrations full directions for work during six grades. II7 pages. Square 8vo. Cloth, $\$ 1.00$. Boards, 60 cts.

Seidel's Industrial Instruction (Smith). A refutation of all objections raised against industrial instruction. 170 pages. $90 \mathrm{cts}$.

\section{Thompson's Educational and Industrial Drawing.}

Primary Free-Hand Series (Nos. 1-4). Each No., per doz., $\$ 1 . \infty$.

Primary Free-Hand Manual. 114 pages. Paper. 40 cts.

Advanced Free-Hand Series (Nos. 5-8). Each No., per doz., \$1.50.

Model and Object Series (Nos. 1-3). Each No., per doz., \$1.75.

Model and Object Manual. 84 pages. Paper. 35 cts.

Esthetic Series (Nos. I-6). Each No., per doz., \$1.50.

Esthetic Manual. 174 pages. Paper. 60 cts.

Mechanical Series (Nos. $1-6$ ). Each No., per doz., \$2.00.

Mechanical Manual. 172 pages. Paper. 75 cts.

Models to accompany Thompson's Drawing :

Set No. I. For Primary Books, per set, $40 \mathrm{cts}$.

Set No. II. For Model and Object Book No. I, per set, oo cts.

Set No. III. For Model and Object Book No. 2, per set, 50 cts.

Thompson's Manual Training, No. I. Treats of Clay Modelling, Stick and Tablet Laying, Paper Folding and Cutting, Color, and Construction of Geometrical Solids. Illustrated. 66 pages. Large 8 vo. Paper. 3o cts.

Thompson's Manual Training, No. 2. Treats of Mechanical Drawing, ClayModelling in Relief, Color, Wood Carving, Paper Cutting and Pasting. Illustrated. 70 pp. Large 8vo. Paper. 3o cts.

Waldo's Descriptive Geometry. A large number of problems systematically arranged, with suggestions. 85 pages. $90 \mathrm{cts}$.

Whitaker's How to Use Wood Working Tools. Lessons in the uses of the universal tools: the hammer, knife, plane, rule, chalk-line, square, gauge, chisel, saw, and auger. 104 pages. $60 \mathrm{cts}$.

Woodward's Manual Training School. Its aims, methods, and results; with detailed courses of instruction in shop-work. Fully illustrated. 374 pages. Octavo. $\$ 2.00$.

Woodward's Educational Value of Manual Training. Sets forth more clearly and fully than has ever been done before the true character and functions of manual training in education. 96 pages. Paper. 25 cts.

Sent postpaid by mail on receipt of price.

D. C. HEATH \& CO., PUBLiSheRS, BOSTON. NEW YORK. CHICAGO. 


\section{SCIENCE.}

Shaler's First Book in Geology. For high school, or highest class in grammar school. \$r.ro. Bound in boards for supplementary reader. $70 \mathrm{cts}$.

Ballard's World of Matter. A Guide to Mineralogy and Chemistry. \$r.o.

Shepard's Inorganic Chemistry. Descriptive and Qualitative; experimental and inductive; leads the student to observe and think. For high schools and colleges. \$r.25.

Shepard's Briefer Course in Chemistry; with Chapter on Organic Chemistry. Designed for schools giving a half year or less to the subject, and schools limited in laboratory facilities. $90 \mathrm{cts}$.

Shepard's Organic Chemistry. The portion on organic chemistry in Shepard's Briefer Course is bound in paper separately. Paper. $30 \mathrm{cts}$.

Shepard's Laboratory Note-Book. Blanks for experiments: tables for the reactions of metallic salts. Can be used with any chemistry. Boards. $40 \mathrm{cts}$.

Benton's Guide to General Chemistry. A manual for the laboratory. 40 cts.

Remsen's Organic Chemistry. An Introduction to the Study of the Compounds of Carbon. For students of the pure science, or its application to arts. \$1.30.

Orndorff's Laboratory Iranual. Containing directions for a course of experiments in Organic Chemistry, arranged to accompany Remsen's Chemistry. Boards. $40 \mathrm{cts}$.

Coit's Chemical Arithmetic. With a short system of Elementary Qualitative Analysir For high schools and colleges. $60 \mathrm{cts}$.

Grabfield and Burns' Chemical Problems. For preparatory schools. 6octs.

Chute's Practical Physics. A laboratory book for high schools and colleges studying pnysics experimentally. Gives free details for laboratory work. \$1.25.

Colton's Practical Zoology. Gives a clear idea of the subject as a whole, by the careful study of a few typical animals. $90 \mathrm{cts}$.

Boyer's Laboratory Manual in Elementary Biology. A guide to the study of animals and plants, and is so constructed as to be of no help to the pupil unless he actually studies the specimens.

Clark's Methods in Microscopy. This book gives in detail descriptions of methods that will lead any careful worker to successful results in microscopic manipulation. $\$ 1.60$.

Spalding's Introduction to Botany. Practical Exercises in the Study of Plants by the laboratory method. $90 \mathrm{cts}$.

Whiting's Physical Measurement. Intended for students in Civil, Mechanical and Electrical Engineering, Surveying, Astronomical Work, Chemical Analysis, Physical Investigation, and other branches in which accurate measurements are required.

I. Fifty measurements in Density, Heat, Light, and Sound. \$1.30.

II. Fifty measurements in Sound, Dynamics, Magnetism, Electricity. \$1.30.

III. Principles and Methods of Physical Measurement, Physical Laws and Principles, and Mathematical and Physical Tables. \$r.3o.

IV. Appendix for the use of Teachers, including examples of observation and reduction. Part IV is needed by students only when working without a teacher. $\$ 1.30$.

Parts I-III, in one vol., \$3.25. Parts I-IV, in one vol., \$4.00.

Williams's Modern Petrography. An account of the application of the micro scope to the study of geology. Paper. $25 \mathrm{cts}$.

For elementary works see our list of books in Elementary Science.

\section{C. HEATH \& CO., PUBLISHERS.}

BOSTON. NEW YORK. CHICAGO. 


\title{
ELEMENTARY SCIENCE.
}

Bailey's Grammar School Physics. A series of inductive lessons in the elements of the science. In press.

Ballard's The World of Matter. A guide to the study of chemistry and mineralogy; adapted to the general reader, for use as a text-book or as a guide to the teacher in giving object-lessons. 264 pages. Illustrated. \$1.00.

Clark's Practical Methods in Microscopy. Gives in detail descriptions of methods that will lead the careful worker to successful results. 233 pages. Illustrated. \$1.60.

Clarke's Astronomical Lantern. Intended to familiarize students with the constellations by comparing them with fac-similes on the lantern face. With seventeen slides, giving twenty-two constellations. $\$ 4.5^{\circ}$.

Clarke's How to find the Stars. Accompanies the above and helps to an acquaintance with the constellations. 47 pages. Paper. 15 cts.

Guides for Science Teaching. Teachers' aids in the instruction of Natural History classes in the lower grades.

I. Hyatt's About Pebbles. 26 pages. Paper. 1o cts.

II. Goodale's A Few Common Plants. 6r pages. Paper. 20 cts.

III. Hyatt's Commercial and other Sponges. Illustrated. 43 pages. Paper. $20 \mathrm{cts}$.

IV. Agassiz's First Lessons in Natural History. Illustrated. 64 pages. Paper. 25 cts.

V. Hyatt's Corals and Echinoderms. Illustrated. 32 pages. Paper. $30 \mathrm{cts}$.

VI. Hyatt's Mollusca. Illustrated. 65 pages. Paper. 30 cts.

VII Hratt's Worms and Crustacea. Illustrated. 68 pages. Paper. 3o cts.

VIII $B$ jatt's Insecta. Illustrated. 324 pages. Cloth. \$r.25.

XII. Crosby's Common Minerals and Rocks. Illustrated. 200 pages. Paper, 40 cts. Cloth, 6o cts.

XIIl Richard's First Lessons in Minerals. 50 pages. Paper. 10 cts.

XIV Bowditch's Physiology. $5^{8}$ pages. Paper. 20 cts.

XV Clapp's 36 Observation Lessons in Minerals. 80 pages. Paper. 30 cts.

XVI Phenix's Lessons in Chemistry. In press.

Pupils Note-Book to accompany No. 15 . $10 \mathrm{cts}$.

Rice's Science Teaching in the School. With a course of instruction in science for the lower grades. $4^{6} \mathrm{pag}$ s. Paper. $25 \mathrm{cts}$.

Ricks's Natural History Object Lessons. Supplies information on plants and their products, on animals and their uses, and gives specimen lessons. Fully illustrated. 332 pages. $\$ 1.50$.

Ricks's Object Lessous and How to Give them.

Volume I. Gives lessons for primary grades. 200 pages. $90 \mathrm{cts}$.

Volume II. Gives lessons tor grammar and intermediate grades. 212 pages. $90 \mathrm{cts}$.

Shaler's First Book in Geology. For high school, or highest class in grammar school. ${ }^{7} 72$ pages. Illustrated. \$1.00.

Shaler's Tencher's Methods in Geology. An. aid to the teacher of Geology. 74 pages. Paper. 25 cts.

Smith's Studies in Nature. A combination of ratural history lessons and language work. 48 pages. Paper. $15 \mathrm{cts}$.

Sent by mail postpaid on receipt of price. See also our list of books in Science.

\section{C. HEATH \& CO., PUBLISHERS,}

\author{
BOSTON. NEW YORK. CHICAGO.
}




\section{EDUCATION.}

Compayré's History of Pedagogy. " The best and most comprehensive history of Education in Euglish." -Dr. G. S. Hall. \$r.75.

Compayré's Lectures on Teaching. "The best book in existence on the theory and practice of education." - Supt. MACALIsTer, Philadelphia. \$1.75.

Compayré's Psychology Applied to Education. A clear and concise statement of doctrine and application on the science and art of teaching. $90 \mathrm{cts}$.

De Garmo's Essentials of Method. A practical exposition of methods with illustrative outlines of common school studies. 65 cts.

De Garmo's Lindner's Psychology. The best Manual ever prepared from the Herbartian standpoint. \$1.0o.

Gill's Systems of Education. "It treats ably of the Lancaster and Bell movement in education, - a very important phase." - Dr. W. T. HARRIS. \$1.25.

Hall's Bibliography of Peđagogical Literature. Covers every department of education. Interleaved, $* 2.00$. \$r.50.

Herford's Student's Froebel. The purpose of this little book is to give young people preparing to teach a brief yet full account of Froebel's Theory of Education. 75 cts.

Malleson's Early Training of Children. "The best book for mothers I ever read." - Elizabeth P. PEABOdy. 75 cts.

Marwedel's Conscious Motherhood. The unfolding of the child's mind in the cradle, nursery and Kindergarten. \$2.00.

Newsholme's School Hygiene. Already in use in the leading training colleges in England. $75 \mathrm{cts}$.

Peabody's Home, Kindergarten, and Primary School. "The best book side of the Bible that I ever rcad." - A Leadinc Teacher. \$r.oo.

Pestalozzi's Leonard and Gertrude. “If we except 'Emile' only, no mor portant educational book has appeared for a century and a half than "Leonard and trude." - The Nation. so cts.

Radestock's Habit in Education. "It will prove a rare 'find' to teachers wh: seeking to ground themselves in the philosophy of their art." - E. H. RUSSELL, W ter Normal School. 75 cts.

Richter's Levana; o:, The Doctrine of Education. "A spirited and sc" bouk." - Prof. W. H. PAYNe. \$I.40.

Rosmini's Method in Education. "The most important pedagogical work writtein." - Thomas Davidson. \$r.5o.

Rousseau's Emile. "Perhaps the most influential book ever written on the sub Education." - R. H. Qurck. go cts.

Methods of Teaching Modern Languages. Papers on the value and on $m$ is of teaching German and French, by prominent instructors. $90 \mathrm{cts}$.

Sanford's Laboratory Course in Physiological Psychology. The rse includes experiments upon the Dermal Senses, Static and Kinæsthetic Senses, 'Taste, Smell, Hearing, Vision, Psychophysic. In Press.

Lange's Apperception: A monograph on Psychology and Pedagogy. Iranslated by the members of the Herbart Club, under the direction of President Charles DeGarmo, of Swarthmore College. \$1.00.

Herbart's Science of Education. Translated by Mr. and Mrs. Felken with a preface by Oscar Browning. \$1.00.

Tracy's Psychology of Childhood. This is the first general treatise covering in a scientific manner the whole field of child psychology. Octavo. Paper. 75 cts.

Sent by mail, postpaid, on receipt of price.

\section{C. HEATH \& CO., PUBLISHERS, BOSTON. NEW YORK. CHICAGO.}



THIS BOOK IS DUE ON THE IAST DATE STAMPED BELOW

AN INITIAL FINE OF 25 CENTS WILL BE ASSESSED FOR FAILURE TO RETURN THIS BOOK ON THE DATE DUE. THE PENALTY WILL INCREASE TO 50 CENTS ON THE FOURTH DAY AND TO \$1.00 ON THE SEVENTH DAY OVERDUE.

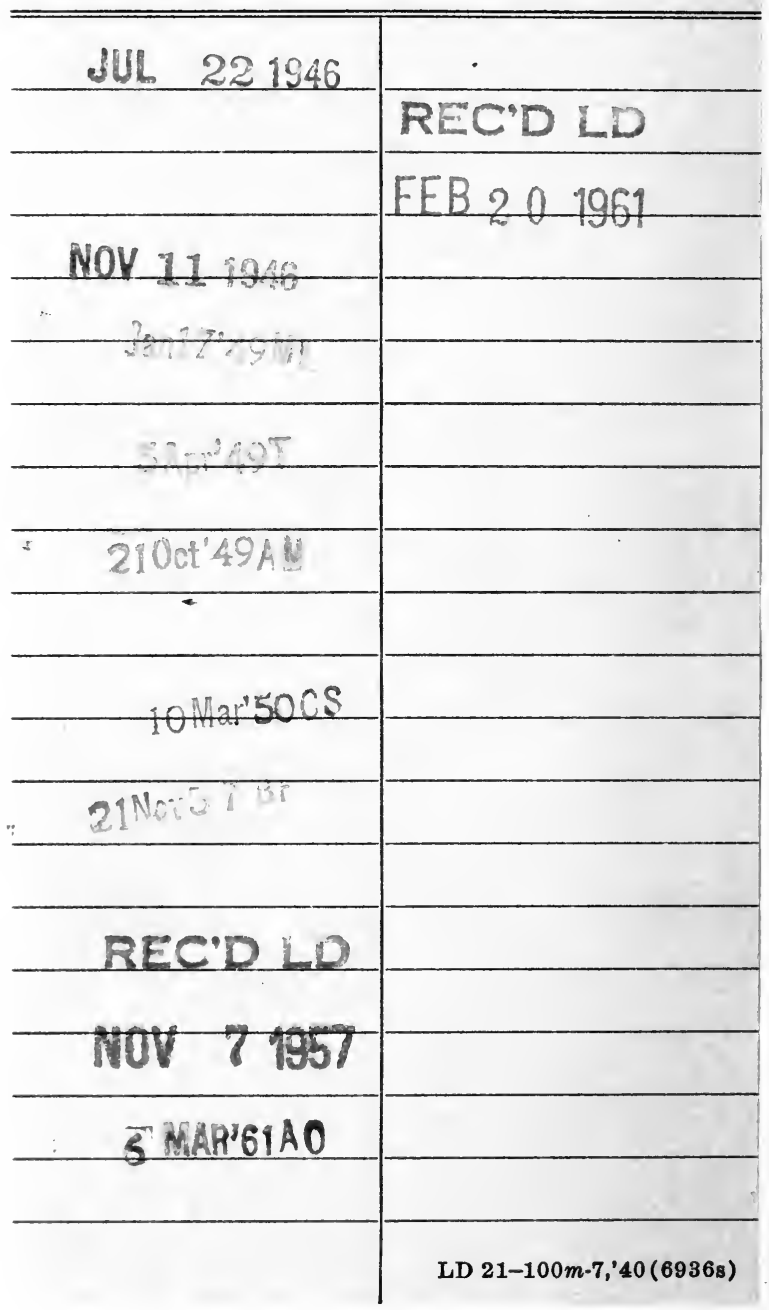




\section{M:206230}

$$
\begin{aligned}
& \text { 2A } 501 \\
& \text { W24 }
\end{aligned}
$$

THE UNIVERSITY OF CALIFORNIA LIBRARY 
